\title{
Chemically active outflow L 1157
}

\author{
R. Bachiller, M. Pérez Gutiérrez ${ }^{\star}$ M. S. N. Kumar, and M. Tafalla \\ IGN Observatorio Astronómico Nacional, Apartado 1143, 28800 Alcalá de Henares, Spain \\ Received 15 January 2001 / Accepted 6 April 2001
}

\begin{abstract}
We present millimeter-wave maps of the L 1157 bipolar outflow in several molecular emission lines. The $\mathrm{CO}$ emission traces the bulk of the outflowing gas in the red and blue shifted lobes displaying a remarkable Sshaped symmetry indicating the presence of a precessing jet. We determine the physical characteristics of the $\mathrm{CO}$ flow and show evidence for 3 or 4 independent episodes of mass ejection from the source. Molecules such as $\mathrm{C}_{3} \mathrm{H}_{2}$, $\mathrm{N}_{2} \mathrm{H}^{+}$and $\mathrm{DCO}^{+}$are seen to be abundant only in the quiescent medium, and result to be the best tracers of the high-density core surrounding the driving source of the outflow. Other molecules $\left(\mathrm{SiO}, \mathrm{CH}_{3} \mathrm{OH}, \mathrm{H}_{2} \mathrm{CO}, \mathrm{HCN}\right.$, $\mathrm{CN}, \mathrm{SO}, \mathrm{SO}_{2}$ ) are abundant in the outflow lobes, but exhibit strong emission gradients. Multiline observations of some species indicate that these gradients are not simply due to excitation effects, but are caused by an actual stratification in the chemical composition of the shocked molecular gas. Shock tracers such as $\mathrm{SiO}, \mathrm{CH}_{3} \mathrm{OH}$, and sulphur-bearing molecules result to be the most promising candidates as potential chemical clocks to study the evolution of outflows. The characteristics of the L 1157 outflow, when compared to those of other outflows from Class 0 sources, indicate that L 1157 is the prototype of a category of bipolar outflows around Class 0 protostars which we denominate "chemically active outflows".
\end{abstract}

Key words. stars: formation - interstellar medium: individual objects: L 1157 - interstellar medium: jets and outflows - interstellar medium: molecules

\section{Introduction}

The L 1157 dark cloud harbors one of the most illustrative cases of a bipolar outflow driven by a Class 0 protostar. Situated at a distance of only $440 \mathrm{pc}$ from the Sun, this flow is highly collimated, and is highly inclined with respect to the line of sight. Thus the overall geometry is very favorable to study the effects of the propagation of the outflow in the surrounding medium. The driving source, identified as L 1157-mm (IRAS 20386+6751) is known to be a low luminosity $\left(11 L_{\odot}\right)$ very young object. The blue lobe of this outflow has been mapped in $\mathrm{CO}$ and $\mathrm{SiO}$ lines using both single dish and interferometric observations (Umemoto et al. 1992; Mikami et al. 1992; Zhang et al. 1995; Gueth et al. 1996; Gueth et al. 1998). The morphology of the shocked regions in a bipolar flow has important implications on the theoretical models. For instance, models for the propagation of a jet into the dense surrounding medium predict the formation of large molecular bow shocks (e.g. Raga \& Cabrit 1993; Stahler 1994). These model predictions are indeed supported by the large bow shocks seen in the $\mathrm{SiO}$ and $\mathrm{CO}$ maps mentioned above and also in the near-IR images at $2.122 \mu \mathrm{m}$

Send offprint requests to: R. Bachiller,

e-mail: bachiller@oan.es

* Present address: Telefónica Investigación y Desarrollo, Laboratorio 226E, c/ Emilio Vargas, 6, 28043 Madrid, Spain. of the $\mathrm{H}_{2}$ emission (Davis \& Eislöffel 1995). We present complete CO maps of the L 1157 outflow and characterize its physical properties.

L 1157 is also well-known for its rich mm-wave spectrum (e.g.: Avery \& Chiao 1996). Bachiller \& Pérez Gutiérrez (1997, hereafter BP97) conducted a deep survey of molecular lines at selected positions in this outflow and found strong enhancement of several molecules in the shocked regions. They noticed that the enhancement factor varied in space both as a function of the species type and of the species abundance. This was the motivation for us to conduct a detailed mapping of this outflow in several molecular lines. The shock heating and rich chemistry in the blue lobe of L 1157 is comparable to that of the Ori KL region. Unlike Ori KL, L 1157 is an isolated source without the confusion of flows from other sources in the surrounding region. It is therefore very advantageous and important to study the shock chemistry in this outflow. We present maps of the L 1157 outflow in selected molecular lines and discuss the evidence for chemical stratification observed in these maps.

\section{Observations and data reduction}

The observations were carried out with the IRAM 30-m radiotelescope at Pico de Veleta (near Granada, Spain) in 1996 October. We used 9 different configurations of 
the three SIS receivers operating at the bands around $\lambda 3 \mathrm{~mm}, 2 \mathrm{~mm}$ and $1.3 \mathrm{~mm}$ to observe at least two molecular lines simultaneously (Table 1 ). The $\mathrm{CO} J=1 \rightarrow 0$ and $J=2 \rightarrow 1$ observations were taken with a single configuration. We observed 320 points covering an area of $2^{\prime} \times 6^{\prime}$ with a spacing of $10^{\prime \prime}$ and an integration time of 20 seconds per position. For the other molecules, we sampled about 80 points in the blue (southern) lobe covering an area of $\sim 1^{\prime} \times 3^{\prime}$ with a spacing of $\sim 10^{\prime \prime}-16^{\prime \prime}$. We also observed the northern lobe in lines of $\mathrm{CH}_{3} \mathrm{OH}$ and $\mathrm{SiO}$. The $\mathrm{SSB}$ receiver temperatures were about $100 \mathrm{~K}$ providing system temperatures in the range $T_{\text {sys }} \sim 300-800 \mathrm{~K}$. The antenna half-power beamwidths and main beam efficiencies ranged from $27^{\prime \prime}$ and 0.75 at $90 \mathrm{GHz}$, to $10^{\prime \prime}$ and 0.37 at $240 \mathrm{GHz}$. This angular resolution corresponds to 4500$12000 \mathrm{AU}$ at the distance of L 1157 ( $\sim 40 \mathrm{pc})$. Pointing was found to be accurate to within $4^{\prime \prime}$. The spectrometers used were filterbanks and autocorrelators providing a spectral resolution up to $0.1 \mathrm{~km} \mathrm{~s}^{-1}$. The observations were made in wobbler switching mode, with the OFF position $4^{\prime}$ from the position of L $1157-\mathrm{mm}\left(\alpha=20^{\mathrm{h}} 39^{\mathrm{m}} 06^{\mathrm{s}} 19\right.$; $\delta=+68^{\circ} 02^{\prime} 15^{\prime \prime} \cdot 9$, J2000), except for the CO lines, which were obtained in position switching. The calibration of the data was achieved by the chopper wheel method. Linear baselines were subtracted from the spectra, and the intensities are reported here in units of main beam brightness temperature.

\section{The CO outflow}

\subsection{Geometry: A point-reflection symmetric outflow}

Figure 1 shows the integrated blue and red shifted ${ }^{12}$ CO $2 \rightarrow 1$ emission from L $1157-\mathrm{mm}$. In agreement with previous work (Umemoto et al. 1992; Zhang et al. 2000), the CO emission is strongly bipolar with respect to L 1157 $\mathrm{mm}$. The western edge of the northern lobe corresponds to a region of strong $\mathrm{H}_{2}$ emission, as seen in the images of Davis \& Eislöffel (1995) and Cabrit et al. (1998). Both outflow lobes are very well aligned on an axis at PA $\sim$ $161^{\circ}$. The terminal projected velocity of the northern lobe is about $65 \%$ higher than that of the southern lobe. Since both lobes are well aligned on the plane of the sky at the same PA, it seems likely that the inclination angles of both lobes to the line of sight are also similar. Therefore, it is very likely that both lobes are well aligned in space, and the observed difference in projected velocity reflects an actual difference in the space velocities of both lobes. This means that the northern lobe would be about $65 \%$ faster than the southern one. This is also consistent with the fact that the northern lobe is more extended than the southern lobe in a way that makes the mean kinematical ages of the two lobes very similar (about $15000 \mathrm{yr}$ ), as expected in the most plausible physical circumstance that both outflow lobes have been created simultaneously. This common factor of space and velocity between the lobes suggests that the outflow is better studied by

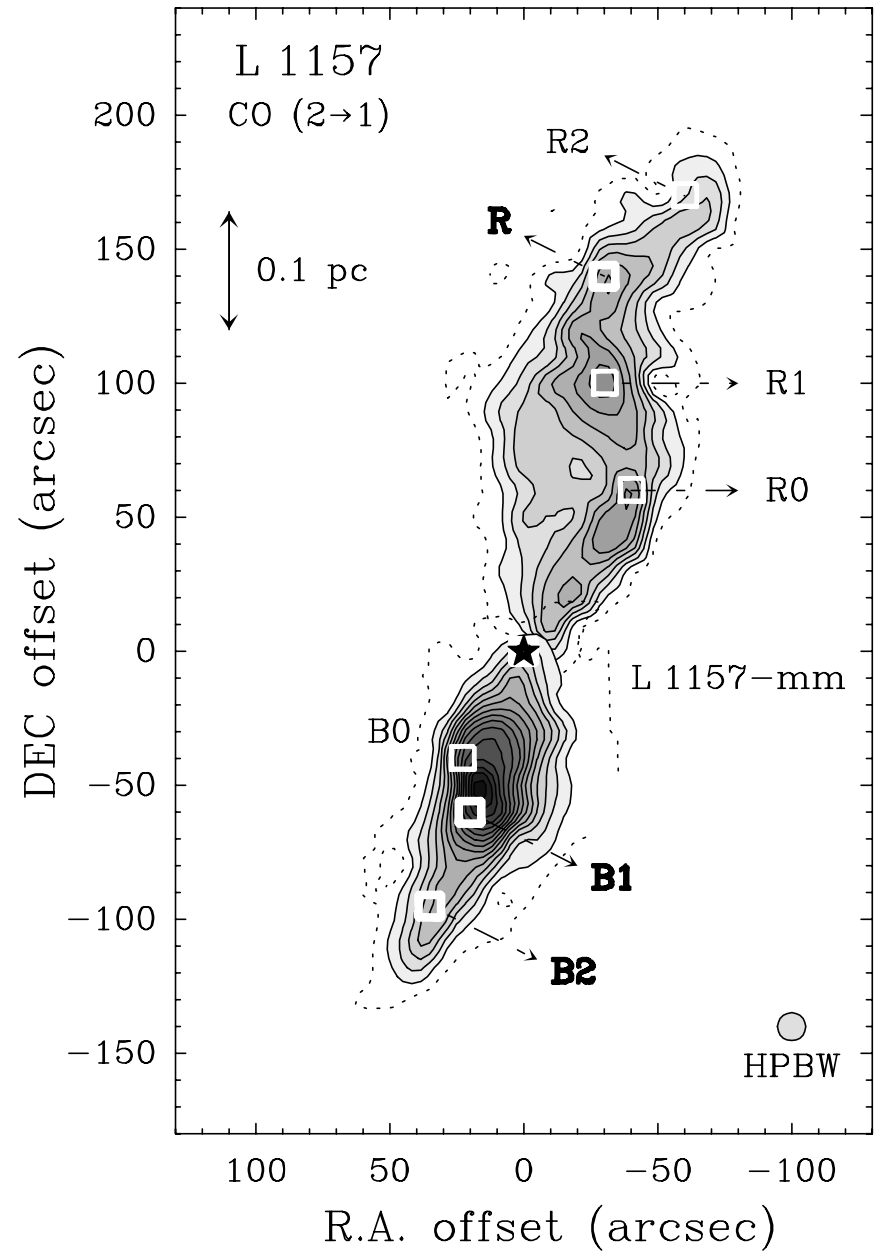

Fig. 1. Integrated emission of $\mathrm{CO}(2 \rightarrow 1)$ showing the bipolar structure of the L 1157 outflow. Velocity intervals are from -20 to $2.7 \mathrm{~km} \mathrm{~s}^{-1}$ for the southern (blue) lobe and from 2.7 to $28 \mathrm{~km} \mathrm{~s}^{-1}$ for the northern (red) lobe. First contour and contour spacing are $11 \mathrm{~K} \mathrm{~km} \mathrm{~s}^{-1}$. The white squares mark the positions studied in Figs. 4 and 5. Boldface labels indicate positions sampled for chemistry by BP97.

comparing velocity intervals spaced accordingly, and such a comparison is presented in Fig. 2.

As seen in Fig. 2, each outflow lobe contains several clumps, and there is a correspondence between clumps in the sense that for each blue clump there is a corresponding red one symmetrically located with respect to the central source (a fact already noted by Zhang et al. 2000 in their $\mathrm{SiO}$ data). This symmetry occurs despite the curved shape of the northern lobe, which seems to be reflected in the southern lobe. The L 1157 outflow, therefore, has a strong point reflection symmetry with respect to L $1157-\mathrm{mm}$, and this symmetry is three-fold as it involves the (curved) lobe shape, the velocity field, and the clump structure. Such a degree of symmetry suggests an intrinsic cause, related more to the central source than to the surrounding environment, although the environment is probably responsible for slowing down the southern lobe and making it smaller. The symmetry in the individual clumps suggests that the outflow has undergone periods of enhanced 
Table 1. Observed molecular transitions.

\begin{tabular}{|c|c|c|c|}
\hline Molecule & Transition & $\nu(\mathrm{GHz})$ & HPBW $\left({ }^{\prime \prime}\right)$ \\
\hline \multicolumn{4}{|l|}{ Blue lobe: } \\
\hline $\mathrm{N}_{2} \mathrm{H}^{+}$ & $1 \rightarrow 0$ & 93.1733505 & 26 \\
\hline $\mathrm{DCO}^{+}$ & $2 \rightarrow 1$ & 144.077321 & 16 \\
\hline $\mathrm{C}^{18} \mathrm{O}$ & $2 \rightarrow 1$ & 219.560319 & 11 \\
\hline $\mathrm{HCN}$ & $1 \rightarrow 0$ & 88.631847 & 28 \\
\hline $\mathrm{SO}$ & $4 \rightarrow 3$ & 138.178648 & 17 \\
\hline $\mathrm{SO}$ & $6 \rightarrow 5$ & 219.949391 & 11 \\
\hline $\mathrm{HCO}^{+}$ & $1 \rightarrow 0$ & 89.188523 & 27 \\
\hline $\mathrm{H}_{2} \mathrm{CO}$ & $2 \rightarrow 1$ & 140.839518 & 17 \\
\hline $\mathrm{H}_{2} \mathrm{CO}$ & $3 \rightarrow 2$ & 225.697772 & 11 \\
\hline $\mathrm{SO}_{2}$ & $3_{13} \rightarrow 2_{02}$ & 104.029416 & 23 \\
\hline $\mathrm{SO}_{2}$ & $5_{15} \rightarrow 4_{04}$ & 135.696011 & 17 \\
\hline $\mathrm{SO}_{2}$ & $11_{111} \rightarrow 10_{010}$ & 221.965210 & 11 \\
\hline $\mathrm{CS}$ & $2 \rightarrow 1$ & 97.980968 & 25 \\
\hline $\mathrm{CS}$ & $3 \rightarrow 2$ & 146.969049 & 16 \\
\hline $\mathrm{CS}$ & $5 \rightarrow 4$ & 244.935606 & 22 \\
\hline $\mathrm{CN}$ & $1, \frac{3}{2} \rightarrow 0, \frac{1}{2}$ & 113.490982 & 22 \\
\hline $\mathrm{CN}$ & $2, \frac{5}{2} \rightarrow 1, \frac{3}{2}$ & 226.874780 & 11 \\
\hline $\mathrm{CN}$ & $2, \frac{3}{2} \rightarrow 1, \frac{1}{2}$ & 226.659543 & 11 \\
\hline \multicolumn{4}{|l|}{ Both lobes: } \\
\hline $\mathrm{SiO}$ & $2 \rightarrow 1$ & 86.846891 & 28 \\
\hline $\mathrm{SiO}$ & $3 \rightarrow 2$ & 130.268702 & 18 \\
\hline $\mathrm{SiO}$ & $5 \rightarrow 4$ & 217.104935 & 11 \\
\hline $\mathrm{CH}_{3} \mathrm{OH}$ & $2_{k} \rightarrow 1_{k}$ & 96.741420 & 25 \\
\hline $\mathrm{C}_{3} \mathrm{H}_{2}$ & $3_{12} \rightarrow 2_{21}$ & 145.089630 & 16 \\
\hline $\mathrm{CH}_{3} \mathrm{OH}$ & $3_{k} \rightarrow 2_{k}$ & 145.103230 & 16 \\
\hline $\mathrm{CH}_{3} \mathrm{OH}$ & $5_{k} \rightarrow 4_{k}$ & 241.791431 & 10 \\
\hline $\mathrm{CO}$ & $1 \rightarrow 0$ & 115.271204 & 21 \\
\hline $\mathrm{CO}$ & $2 \rightarrow 1$ & 230.537990 & 11 \\
\hline
\end{tabular}

ejection, and the curved shape suggests that there have been variations in the direction of the driving wind. In Sect. 5 we will explore these ideas with the help of a simplified model.

To further illustrate the symmetry of the outflow lobes (once the space/velocity factor is taken into account), we present in Fig. 3 a position-velocity diagram along the outflow axis. The clumps B0, B1, and B2, seem related, respectively, to $\mathrm{R} 0, \mathrm{R} 1$, and $\mathrm{R} 2$. Kinematical ages for the R0-B0 pair are $4500 \mathrm{yr}$, for the B1-R1 pair are $7000 \mathrm{yr}$, and for the B2-R2 pair are 15000 yr. Figure 3 shows that the events B1 and R1 have larger velocities (almost twice) compared to the other two events B0, B2 and R0, R2. This fact can also be noticed in the $\mathrm{SiO}$ data of the blue lobe from Gueth et al. (1996, hereafter referred to as GGB) and of the red lobe by Zhang et al. (2000). This result can be due to two possible reasons. The second events (B1, R1) follows the first events (B2, R2) in phase (by morphology) and therefore moves into a cavity that is already excavated by the first events, resulting in lesser obstruction and therefore higher velocity. Alternatively, this could be due to varying velocity of the jet in time (see, e.g., Reipurth \& Raga 1999).

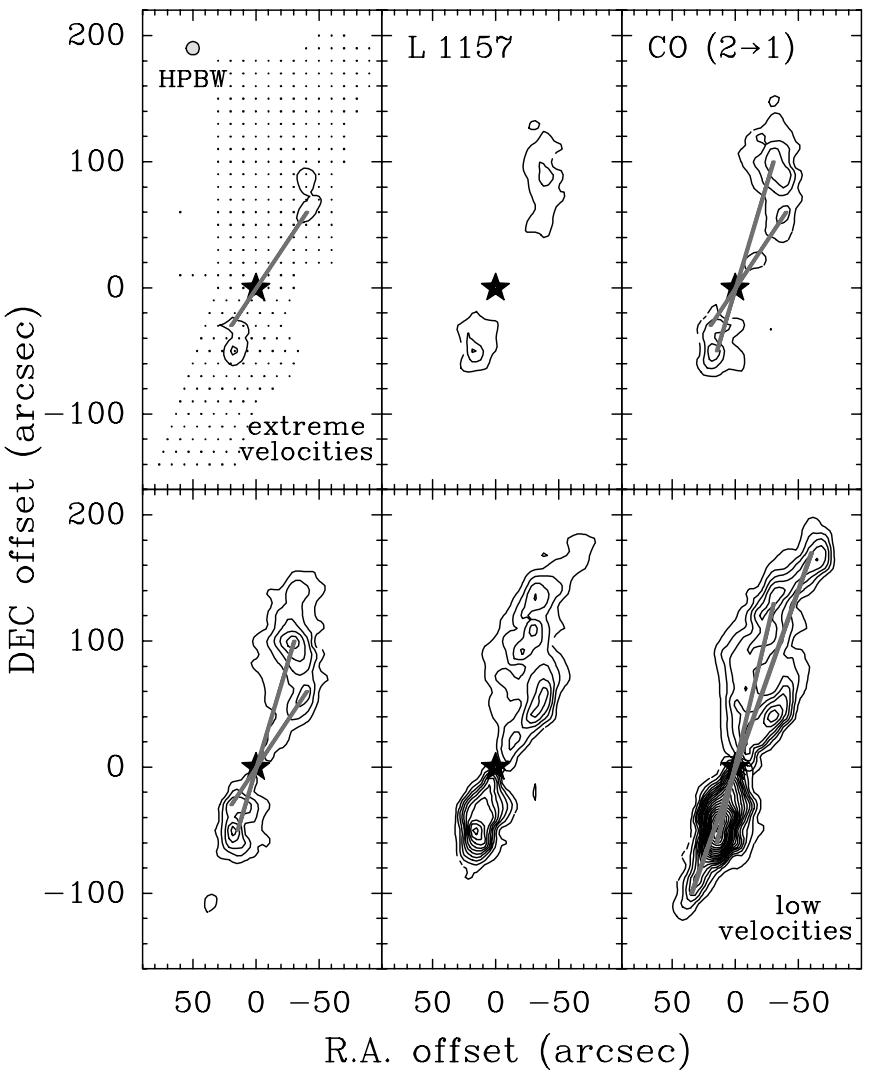

Fig. 2. CO emission in several selected velocity intervals. Each panel represents a velocity increase of $2.5 \mathrm{~km} \mathrm{~s}^{-1}$ for the blue lobe (South) and $4 \mathrm{~km} \mathrm{~s}^{-1}$ for the red one (North) with respect to the central velocity of the quiescent emission $\left(V_{\mathrm{LSR}}=\right.$ $\left.2.7 \mathrm{~km} \mathrm{~s}^{-1}\right)$. First contour and step are $2.5 \mathrm{~K} \mathrm{~km} \mathrm{~s}^{-1}$ for the southern lobe and $4 \mathrm{~K} \mathrm{~km} \mathrm{~s}^{-1}$ for the northern one. Thick lines mark the axis of the outflow for each episode of ejection.

\subsection{Physical parameters of the outflow}

We first estimate the ${ }^{12} \mathrm{CO}(2 \rightarrow 1)$ optical depth measuring the ${ }^{12} \mathrm{CO} /{ }^{13} \mathrm{CO}$ ratio toward selected positions. To compute the value of this ratio (as well of that of the CO $2 \rightarrow 1 / 1 \rightarrow 0$ ratio, see below) we have first smeared the $2 \rightarrow 1$ data to the angular resolution of the $1 \rightarrow 0$ data $\left(\sim 21^{\prime \prime}\right)$. In Fig. 4 we present the result for B0, one of the brightest blue outflow positions (see Fig. 1), showing that the ratio is close to 1 at ambient velocities (high optical depth), but increases rapidly in the outflow regime and stabilizes near 40 for velocities bluer than $V_{\mathrm{LSR}} \sim 0.5 \mathrm{~km} \mathrm{~s}^{-1}$. This value is close to the isotopic ratio (which is uncertain given isotopic fractionation, see Wilson \& Rood 1994), so we consider it representative of the optically thin regime. For the red lobe (not shown in Fig. 4), a similar situation is reached for velocities larger than $V_{\mathrm{LSR}} \sim 5 \mathrm{~km} \mathrm{~s}^{-1}$. In the following analysis, we will concentrate on this optically thin outflow regime, ignoring the highly uncertain optically thick part.

For optically thin $\mathrm{CO}$ emission, the $2 \rightarrow 1 / 1 \rightarrow 0$ ratio $\left(R_{21}\right)$ is a good indicator of the $\mathrm{CO}$ excitation temperature, which is expected to be close to the gas kinetic temperature due to the low dipole moment of the molecule. 


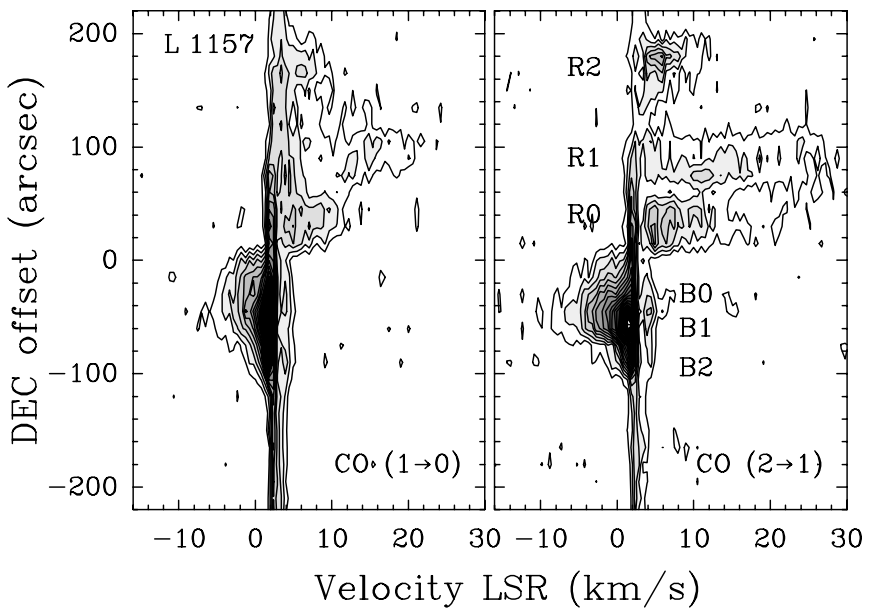

Fig. 3. Position-velocity diagrams of the $\mathrm{CO}$ emission along the L 1157 outflow. First contour and step are $5 \%$ of the maximum, and the spectral resolution is $0.5 \mathrm{~km} \mathrm{~s}^{-1}$. The labels indicate the positions shown in Fig. 1.

In Fig. 5 we present $R_{21}$ towards the positions marked in Fig. 1, showing that it varies with position. Near the protostar and in most of the northern lobe $R_{21} \sim 2$, indicating an excitation temperature of $\sim 15 \mathrm{~K}$, while at the lobe end the ratio increases to $\sim 2.7$, indicating higher temperatures, of the order of $25-30 \mathrm{~K}$. In the southern lobe, on the other hand, ratios as high as 3.5 (indicating temperatures of $80 \mathrm{~K}$ ) are seen toward B1, while at the tip of the lobe temperatures are of the order of $25 \mathrm{~K}$. These values are in good agreement with the $\mathrm{NH}_{3}$ measurements of Tafalla \& Bachiller (1995), and seem not to depend on the velocity of the outflowing gas. The largest temperatures in the $\mathrm{CO}$ outflow are most likely due to gas heating by outflow shocks, as previously suggested by Umemoto et al. (1992) and Bachiller et al. (1993).

Given the above temperatures and the fact that the outflow regime is optically thin in $\mathrm{CO}$ for velocities smaller than $0.5 \mathrm{~km} \mathrm{~s}^{-1}$ and larger than $5 \mathrm{~km} \mathrm{~s}^{-1}$, we calculate the outflow mass, momentum, and energy. We assume a CO abundance of $10^{-4}$ and apply standard techniques (see e.g. Margulis \& Lada 1985). The results are presented in Table 2. It is appropriate to remind here that estimates of outflow physical parameters from CO observations are subject to important sources of uncertainty. As concerns the mass, the main uncertainties arise (i) in the placement of the velocity boundary between the high velocity wing and the ambient line and (ii) in the assumed kinetic temperature (note that the mass estimate is nearly proportional to the assumed temperature). We believe that our estimate of the $\mathrm{L} 1157$ outflow mass $\left(\sim 0.6 M_{\odot}\right)$ is accurate within a factor of 2 . With regard to the flow momentum and energy, the estimates depend critically on the knowledge of the inclination of the flow axis to the line of sight. The values given in Table 2 are not corrected for projection effects, and the corresponding correction factors are quite important in the case of the L 1157 outflow owing to its high inclination with respect to the line of sight

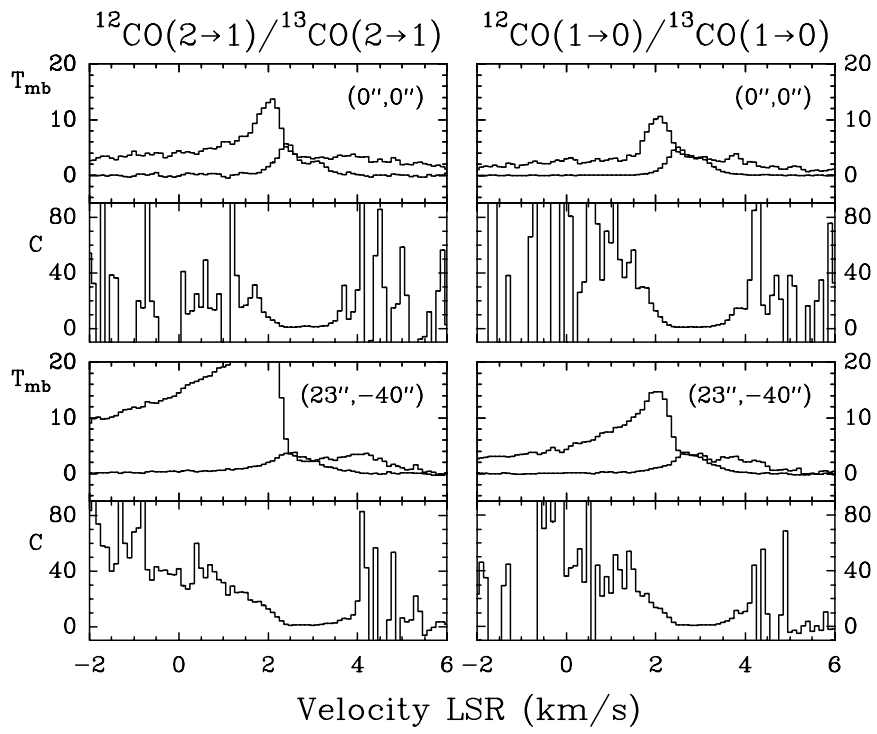

Fig. 4. Profiles of the ${ }^{12} \mathrm{CO}$ and ${ }^{13} \mathrm{CO}$ lines and their ratio towards the central position and the southern lobe (B0) of L 1157. The spectra are convolved to the same spectral and spatial resolution. For B0, the line ratio raises to values $\sim 40$ for velocities bluer than $0.5 \mathrm{~km} \mathrm{~s}^{-1}$ indicating the beginning of the optically thin regime.

(as indicated by the small spatial overlap of both outflow lobes around the exciting source). We have estimated that if this inclination angle is $81^{\circ}$, as suggested by GGB, the momentum and the kinetic energy reported in Table 2 should be multiplied by factors of 6.4 and 41 , respectively. We finally point out that model simulations by Cabrit \& Bertout (1990) (see also Margulis \& Lada 1985) that the most accurate methods to derive outflow parameters from CO observations estimate the mass within a factor of $\sim 2$, the momentum within a factor of $\sim 5$, and the kinetic energy within a factor of $\sim 10$ (providing that the momentum and energy have been corrected for projection effects).

\section{Chemical tracers of the outflow}

\subsection{The southern lobe}

In Fig. 6 we present maps of the southern lobe obtained in the emission of molecules other than CO. Though we have observed more than one line per molecule, in this figure we present only those with the best $\mathrm{S} / \mathrm{N}$. The squares mark the positions from BP97 where the survey of the molecular lines have been presented. It can be immediately seen that there is a strong gradient in the distribution of emission among different species along the outflow lobe. Molecules such as $\mathrm{C}_{3} \mathrm{H}_{2}, \mathrm{DCO}^{+}$and $\mathrm{N}_{2} \mathrm{H}^{+}$trace only the central condensation (along the axis of a hypothetical disk) and are not observed in the outflow or the quiescent medium. In contrast, other molecules such as $\mathrm{SiO}, \mathrm{HCN}, \mathrm{H}_{2} \mathrm{CO}$, $\mathrm{CS}, \mathrm{CH}_{3} \mathrm{OH}, \mathrm{SO}$ and $\mathrm{SO}_{2}$ follow a striking segregation tendency. The emission from $\mathrm{HCO}^{+}$and $\mathrm{CN}$ is present only between the protostar and the first peak B1. The structure of this region can be seen more clearly in $\mathrm{H}_{2} \mathrm{CO}$, 


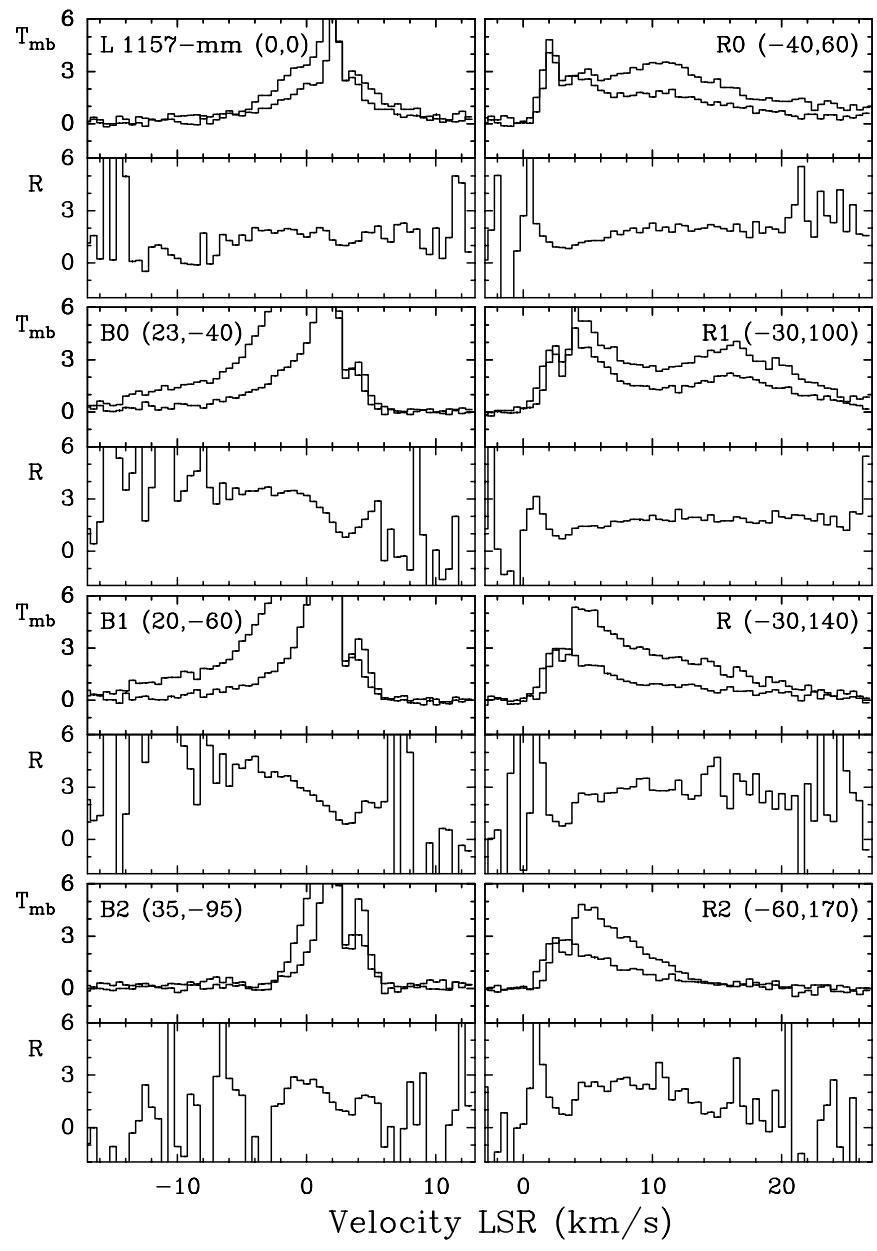

Fig. 5. ${ }^{12} \mathrm{CO} J=2 \rightarrow 1$ and $1 \rightarrow 0$ spectra and their ratio towards several positions in the L 1157 outflow. The spectra are convolved to the same spatial and spectral resolution. Numbers in parenthesis are offsets from L $1157-\mathrm{mm}$ in arcseconds. In the outflow wings line ratios range from 3.5 towards B1 to about 2 towards R1. For an optically thin case, these ratios indicate temperatures of about $80 \mathrm{~K}$ for $\mathrm{B} 1$ and about $15 \mathrm{~K}$ for $\mathrm{R} 1$.

$\mathrm{CS}, \mathrm{CH}_{3} \mathrm{OH}$, and $\mathrm{SO}$ molecules. The structure around the B2 peak is best seen in the maps of $\mathrm{SiO}, \mathrm{CH}_{3} \mathrm{OH}$, and especially in $\mathrm{SO}$ and $\mathrm{SO}_{2}$ molecules.

These gradients in the molecular line distributions may be due to a real segregation in the abundances of the chemical species or to mere excitation effects. In order to distinguish between these two possibilities, we have obtained maps of the emission of several rotational lines of the same species, which can trace regions of different temperature due to their different excitation energies. In Fig. 7 we show the maps of the $J=2 \rightarrow 1,3 \rightarrow 2$, and $5 \rightarrow 4$ emission lines of $\mathrm{SiO}, \mathrm{CH}_{3} \mathrm{OH}$, and $\mathrm{CS}$. We can see that, after taking into account the differences in the angular resolution of the maps, the distribution of the emission is very similar for the transitions of low and high excitation energies. Though the effect of different spatial resolution is very obvious, the positions of the emission peaks are almost the same. This is clear evidence that the the distribution gradients of the emission are not due to simple
Table 2. Physical parameters of the bipolar outflow L 1157 obtained from the $\mathrm{CO}$ observations. Velocity intervals are in $\mathrm{km} \mathrm{s}^{-1}$ (LSR). Estimates of momentum $(P)$ and kinetic energies $\left(E_{\mathrm{k}}\right)$ are not corrected for projection effects (see text for discussion on the uncertainties on the parameter estimates).

\begin{tabular}{lccc}
\hline \hline Blue lobe & $\begin{array}{c}M \\
\left(M_{\odot}\right)\end{array}$ & $\begin{array}{c}P \\
\left.\mathrm{~km} \mathrm{~s}^{-1}\right)\end{array}$ & $\begin{array}{c}E_{\mathrm{k}} \\
\left(10^{44} \mathrm{erg}\right)\end{array}$ \\
\hline low $v(-8.5$ to -0.5$)$ & 0.32 & 0.91 & 0.42 \\
high $v(-17.5$ to -8.5$)$ & 0.03 & 0.50 & 0.73 \\
extreme $v(-26.5$ to -17.5$)$ & 0.01 & 0.25 & 0.62 \\
\hline total blue wing & 0.36 & 1.66 & 1.77 \\
\hline \multicolumn{4}{c}{} \\
\hline Red lobe & $M$ & $P$ & $E_{\mathrm{k}}$ \\
& $\left(M_{\odot}\right)$ & $\left(M_{\odot} \mathrm{km} \mathrm{s}^{-1}\right)$ & $\left(10^{44} \mathrm{erg}\right)$ \\
\hline low $v$ (5 to 14$)$ & 0.18 & 0.76 & 0.02 \\
high $v(14$ to 23$)$ & 0.06 & 0.91 & 0.53 \\
extreme $v(23$ to 32$)$ & 0.02 & 1.38 & 1.31 \\
\hline total red wing & 0.26 & 3.05 & 1.86 \\
\hline \hline
\end{tabular}

excitation effects but mainly due to an actual segregation of the molecular species.

In order to confirm that the differences in line emission reflect actual differences in chemical abundances, we have carried out radiative transfer calculations using a Large-Velocity-Gradient (LVG) code to interpret the observations of molecular species for which we have observed more than a rotational line, namely $\mathrm{SiO}, \mathrm{CS}$, and $\mathrm{CN}$. The case of $\mathrm{CN}$ is particularly complex because of its fine and hyperfine molecular structure, and because the collisional rates are unknown. In our calculations, we considered that the hyperfine components of each rotational transitions are completely coincident, and that the fine components present no line overlap. The $\mathrm{CN}$ collisional rates were estimated from those of CS (Turner et al. 1992) corrected for the different molecular sizes, and we assumed the infinite sudden (IOS) approximation to account for the fine structure. Although such rates are clearly inadequate to study details of the CN collisional excitation, they should be approximate enough for the estimates of the rotational temperature. The values of rotational temperatures of $\mathrm{CH}_{3} \mathrm{OH}$ were computed by using the "rotation diagram method" (e.g. Cummings et al. 1986) at some selected positions of the L 1157 southern lobe.

Although LVG calculations provide estimates of the excitation temperature and the line opacity, it is not possible to separate the effects on the excitation of the gas density and temperature. Therefore, some additional independent estimates of these parameters are necessary. Estimates of the kinetic temperature have been previously obtained at different outflow positions using multiline $\mathrm{NH}_{3}$ observations (Bachiller et al. 1993; Tafalla \& Bachiller 1995) and CO observations (see Sect. 3.2). Estimates of the densities were obtained from the methanol rotational temperatures by comparing to detailed radiative transfer models as proposed by Bachiller et al. (1998, their Fig. 9).

As a result of these calculations we find that only moderate changes in the physical conditions (density and 


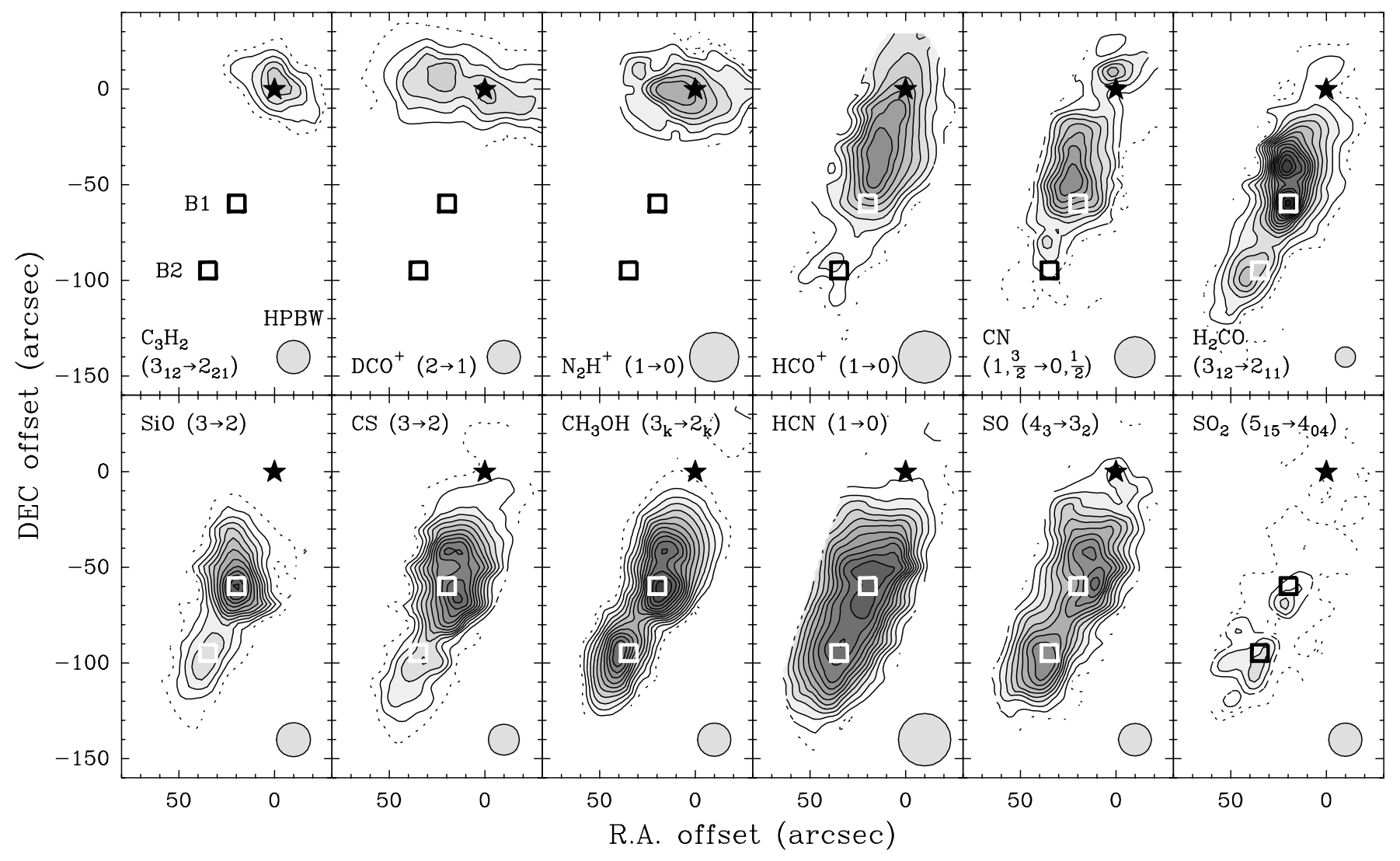

Fig. 6. Maps of the molecular emission towards the southern lobe of L 1157. First contour and step are $0.07 \mathrm{~K} \mathrm{~km} \mathrm{~s}^{-1}$ for $\mathrm{C}_{3} \mathrm{H}_{2}$, 0.3 for $\mathrm{DCO}^{+}, 0.8$ for $\mathrm{N}_{2} \mathrm{H}^{+}, 1.3$ for $\mathrm{HCO}^{+}$and $\mathrm{SO}, 0.7$ for $\mathrm{CN}, 2$ for $\mathrm{H}_{2} \mathrm{CO}$, CS and $\mathrm{SiO}, 6$ for $\mathrm{CH}_{3} \mathrm{OH}$, and $0.5 \mathrm{~K} \mathrm{~km} \mathrm{~s}-1$ for $\mathrm{SO}_{2}$. The star symbol marks the position of the Class 0 protostar L 1157-mm, and the squares mark the positions where an initial molecular survey was carried out (BP97). Note the striking differences in the line emission distribution which are discussed in the text.

temperature) take place across the shocked regions sampled by the molecular species mentioned above ( $\mathrm{SiO}, \mathrm{CS}$, $\mathrm{CN}, \mathrm{CH}_{3} \mathrm{OH}$; see also Sect. 5.2). The kinetic temperatures in the outflow range from 40 to $100 \mathrm{~K}$. These values are in general agreement with very recent sub-mm observations (Hirano \& Taniguchi 2001) showing that the region sampled by the CO $J=6-5,4-3,3-2$ lines has temperatures ranging from 50 to $170 \mathrm{~K}$, and with observations of the very highly excited $(5,5)$ and $(6,6)$ metastable inversion lines of $\mathrm{NH}_{3}$ (Umemoto et al. 1999), which provide values of the kinetic temperatures in the range 40 to $140 \mathrm{~K}$. Densities in this blueshifted lobe are in the range of a few $10^{4}$ to a few $10^{5} \mathrm{~cm}^{-3}$. Indeed the uncertainties in these LVG estimates are quite large. We believe that temperatures are accurate within $30 \%$, and densities within a factor of 10 . In any event, the moderate differences found in physical conditions cannot explain alone the large changes observed in the emission from the different molecules, which confirms that important variations in the chemical abundances take place along the outflow.

In Fig. 6, notice that $\mathrm{H}_{2} \mathrm{CO}, \mathrm{CS}, \mathrm{CH}_{3} \mathrm{OH}$, and $\mathrm{SO}$ molecules (maps of higher frequencies) display a prominent peak of emission that is marked as B0 in Fig. 1. This peak, which was not clearly seen in some previous maps of $\mathrm{CO}$ and $\mathrm{SiO}$, is unambiguously identified here at a position offset of $\left(23^{\prime \prime},-40^{\prime \prime}\right)$ from the central protostar. Its position, just north of B1, is consistent with the idea of a underlying precessing jet, and also compatible with the concept of a cavity wall excavated by the propagation of the shock B1 (GGB, Gueth et al. 1998). This peak is indeed the first peak seen in the position-velocity diagram of $\mathrm{SiO}$ emission by GGB. The kinetic temperature we found for this position is $\sim 40 \mathrm{~K}$, significantly lower than the $80 \mathrm{~K}$ at B1. This trend is in good agreement with estimates by Hirano \& Taniguchi (2001).

In Fig. 8 we present the velocity channel maps of the emission from some molecular lines with best $\mathrm{S} / \mathrm{N}$ ratio. Conforming to the CO maps of Fig. 2, we see that the bulk of the emission is blue shifted with respect to the systemic velocity $\left(2.7 \mathrm{~km} \mathrm{~s}^{-1}\right)$. Peak B1 dominates the high velocities, whereas the emission from B2 is concentrated at the lowest velocities. B0 presents an intermediate velocity, and it is particularly prominent in the $\operatorname{CS}(3 \rightarrow 2)$ maps. This line also shows appreciable emission at the highest velocities, whereas $\mathrm{HCO}^{+}$is seen only at moderately low velocities. Such differences in the terminal velocities of the different species is further illustrated in the positionvelocity diagrams of the blue lobe along the outflow axis $\left(\mathrm{PA}=161^{\circ}\right)$ presented in Fig. 9. We note that most of the emission takes place at velocities close to that of the 


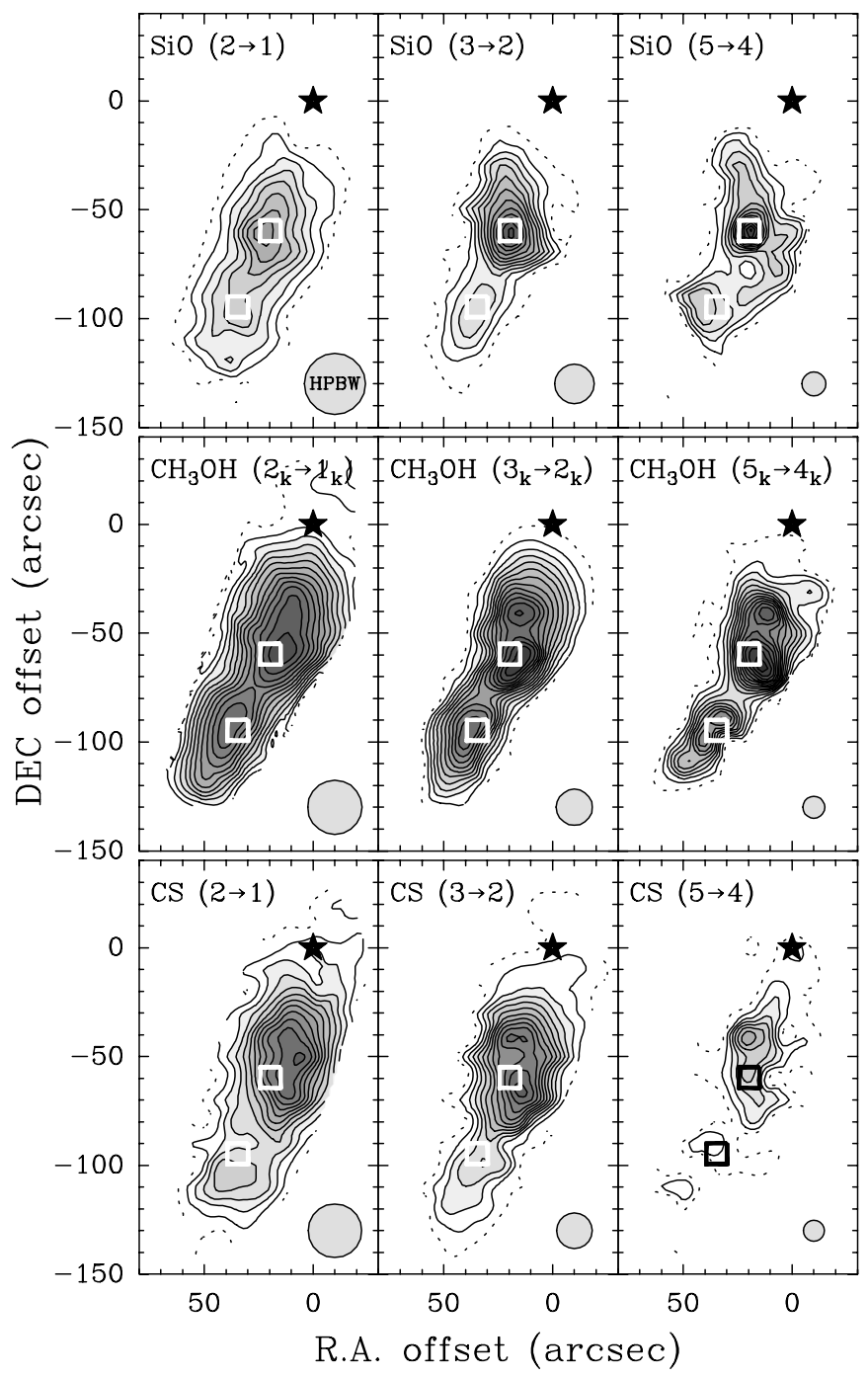

Fig. 7. Maps of the integrated emission for several transitions of $\mathrm{SiO}, \mathrm{CH}_{3} \mathrm{OH}$, and CS. First contour and step are 2, 2 and $1.5 \mathrm{~K} \mathrm{~km} \mathrm{~s}^{-1}$ for $\mathrm{SiO} 2 \rightarrow 1,3 \rightarrow 2$, and $5 \rightarrow 4$, respectively; 2.5, 5 and $5 \mathrm{~K} \mathrm{~km} \mathrm{~s}^{-1}$ for the $\mathrm{CH}_{3} \mathrm{OH}$ lines; and 1.5, 2 and $2.5 \mathrm{~K} \mathrm{~km} \mathrm{~s}^{-1}$ for the CS lines.

ambient gas indicating that most of the material moves at low velocities. However, $\mathrm{CO}$ and $\mathrm{SiO}$ reach terminal velocities which are significantly higher than those of $\mathrm{HCO}^{+}$, $\mathrm{H}_{2} \mathrm{CO}$, SO and CS. This effect could be related to differences in the mechanisms of formation of the different species.

\subsection{The northern lobe}

In our previous molecular survey (BP97) we saw that the emission from the northern lobe of L 1157 is much weaker than that from the southern lobe. However, we have explored the red lobe of L 1157 and detected emission from $\mathrm{SiO}$ and $\mathrm{CH}_{3} \mathrm{OH}$ at several regions along the outflow. In Fig. 10 we present the $\lambda 2 \mathrm{~mm}$ maps of $\mathrm{SiO}$ and $\mathrm{CH}_{3} \mathrm{OH}$. The squares are the same positions as shown in the integrated CO map of Fig. 1.
The most evident feature in these maps is the large asymmetry between the emission of the northern and southern lobe, even more pronounced than in the $\mathrm{CO}$ maps. The emission in the north is concentrated in some discrete and separated locations. These peaks are common to the emission from $\mathrm{CH}_{3} \mathrm{OH}, \mathrm{SiO}$ and also $\mathrm{CO}$, but the relative intensities are different. $\mathrm{SiO}$ peaks far away from the protostar whereas the emission from $\mathrm{CH}_{3} \mathrm{OH}$ is present in nearer points, probably related to more recent ejection events. The emission of $\mathrm{CH}_{3} \mathrm{OH}$ is too weak, but that of $\mathrm{SiO}$ can be separated into velocity channels, as shown in Fig. 11. We can see that the emission farther from the central source have lower velocities than those close to the source. This is consistent with the results of the $\mathrm{CO}$ maps in Fig. 5. We can also match the different episodes of ejection in the northern and southern lobes. As in the case of Fig. 3 we have used a scale factor between the two lobes to enable a direct comparison, owing to the asymmetry in size and velocity of the lobes. The velocity intervals are the same as in Fig. 3. This map reconfirms the change in the direction of the ejection from one episode to the other.

\section{Discussion}

\subsection{A precessing outflow}

As mentioned in Sect. 3.1, the L 1157 outflow presents a clear point reflection symmetry in both position and velocity, with a one to one correlation between the blue spots $\mathrm{B} 0, \mathrm{~B} 1$, and $\mathrm{B} 2$ and the red peaks $\mathrm{R} 0, \mathrm{R} 1$, and $\mathrm{R} 2$ (see $\mathrm{CO}$ and $\mathrm{SiO}$ maps in Figs. 1, 2, 10, and 11). This point reflection symmetry suggests that the outflow has precessed over its lifetime, and that the bright peaks correspond to events of enhanced ejection. These ideas have been previously explored by GGB, who modeled the southern CO lobe with two broad bow shocks resulting from a precessing jet. The shocks in the GGB model were located at the B1 and B2 positions, while the B0 peak was considered to be part of the B1 bow shock. As B1 and B2 are almost aligned with L $1157-\mathrm{mm}$, the precession angle these authors estimate is very small $\left(6^{\circ}\right)$. More recently, Zhang et al. (2000) have presented $\mathrm{CO}$ and $\mathrm{SiO}$ maps of both lobes and suggested that the B0 peak represents an independent ejection (due to its pairing with $\mathrm{R} 0$ ), implying a larger precession angle $\left(\sim 15^{\circ}\right)$ and a narrower bow shock structure. Our observations support this second interpretation, and to further explore this scenario, we attempt to reproduce the outflow geometry with a simple, geometrical model of a narrow, precessing jet.

Our model assumes that the jet moves in a straight line with constant velocity, and that the ejection direction rotates with constant angular speed. We fit the asymmetry between the blue and red lobes (Sect. 3.1) assuming that the blue outflow velocity is 0.65 times the velocity in the red lobe, and vary the outflow age and rotation period to fit the positions of the bright outflow $\mathrm{SiO}$ spots. We assume a precession angle of $15^{\circ}$, in agreement with 


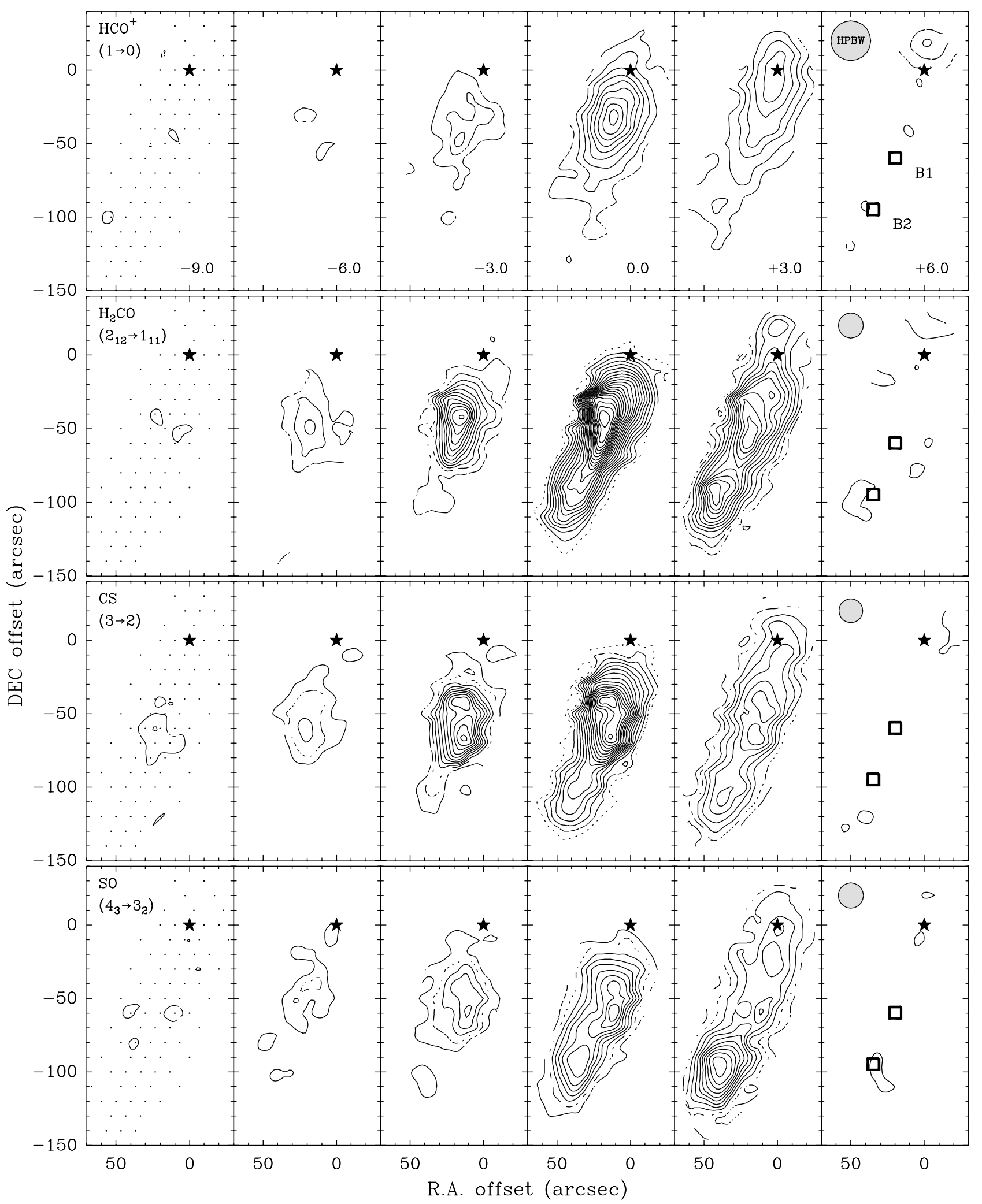

Fig. 8. Channel maps of the molecular emission in the southern lobe of the L 1157 outflow. The emission has been integrated over $3 \mathrm{~km} \mathrm{~s}^{-1}$ intervals centered around the LSR velocity marked in the top row (lower-right corner of each panel). The second panel from the right is centered at $V_{\mathrm{LSR}}=3 \mathrm{~km} \mathrm{~s}^{-1}$, which corresponds roughly to the peak velocity of the central condensation $\left(V_{\mathrm{LSR}} \sim 2.7 \mathrm{~km} \mathrm{~s}^{-1}\right)$, so the bulk of the observed emission in this region is blue shifted. First contour and step are $0.7 \mathrm{~K} \mathrm{~km} \mathrm{~s}^{-1}$ for $\mathrm{HCO}^{+}$and $\mathrm{H}_{2} \mathrm{CO}$, and $0.6 \mathrm{~K} \mathrm{~km} \mathrm{~s}^{-1}$ for CS and SO. Symbols are as in Fig. 6, and the squares in the right panels mark the B1 and $\mathrm{B} 2$ positions. Note again the differences in the distribution of the different line emissions. Extreme cases are $\mathrm{HCO}^{+}$, which shows peaks at relatively low velocities and close to the protostellar position, and SO, which shows higher terminal velocities and strong peaks far away from the protostar. The $\mathrm{H}_{2} \mathrm{CO}$ and $\mathrm{CS}$ lines exhibit an intermediate behavior. 


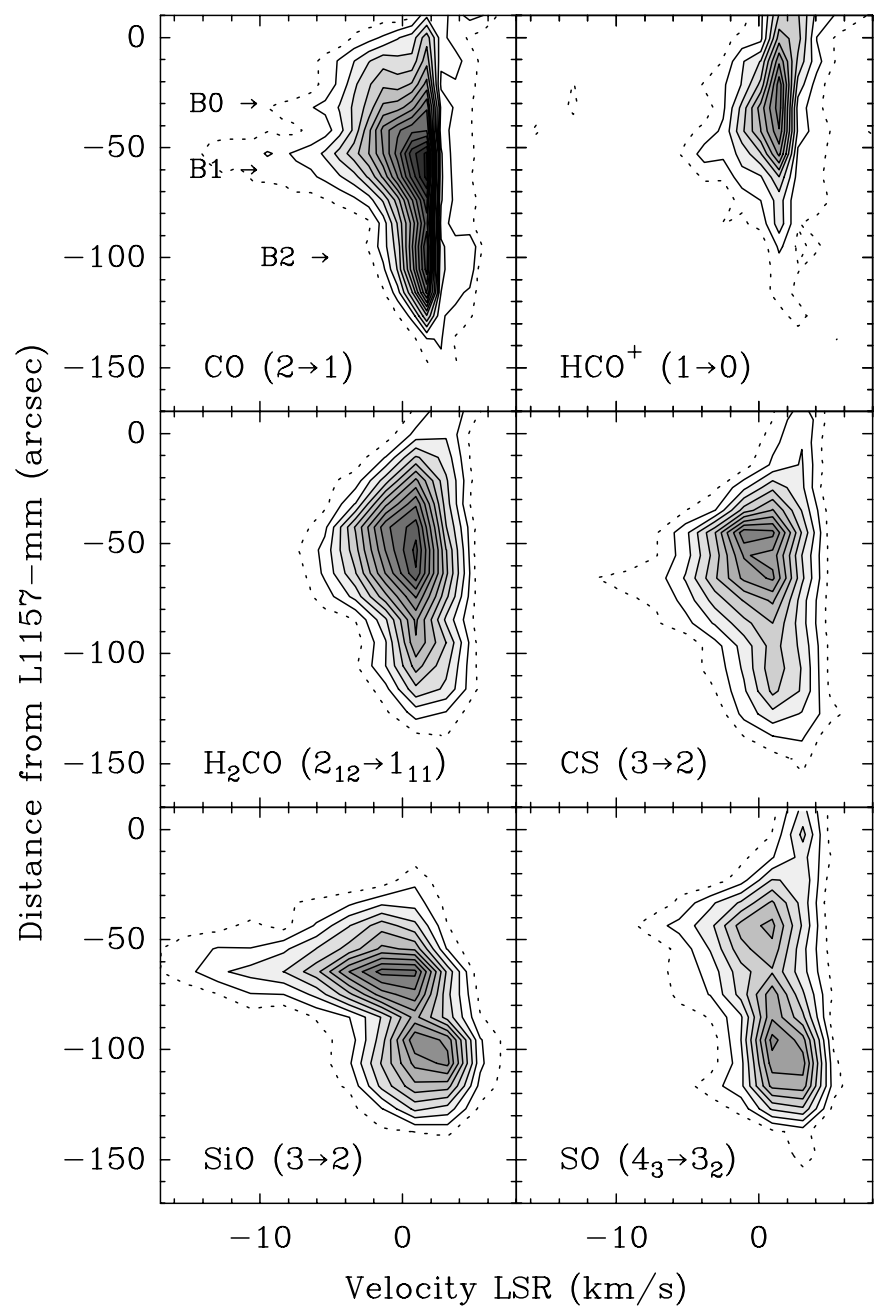

Fig. 9. Position-velocity diagrams of the southern lobe of the L 1157 outflow in several strong emission lines. First contour and step are $2 \mathrm{~K}$ for $\mathrm{CO}, 0.3$ for $\mathrm{CS}, 0.4$ for $\mathrm{H}_{2} \mathrm{CO}$, and 0.25 for $\mathrm{SiO}$ and $\mathrm{HCO}^{+}$, in the $T_{\mathrm{mb}}$ scale. The three emission peaks (B0, B1, and B2) that we identify as corresponding to different episodes of ejection are indicated in the first panel.

Zhang et al. (2000), and present the result of the modeling in Fig. 12.

As it can be seen in the left panel of Fig. 12, the simple model fits rather well the position of the $\mathrm{SiO}$ emission, both the bright peaks and the extended part. This good fit shows that the location of the most recently shocked material is consistent with the expected position of the working surface of a precessing jet. If this model is correct, the outflow time scale should be approximately equal to one precession period, which can also be seen from the fact that the northern lobe forms a single sinusoidal wave. This characteristic sets L 1157 apart from other flows where precession has been proposed, like HH333 (Bally et al. 1996), HH34, RNO43 (Eislöffel \& Mundt 1997) and PV Cep (Gómez et al. 1997). In all these cases, the jet/flow bends only once, giving them a global S-shape, irrespective of their sizes and dynamical ages. The repetitive S-shape of L 1157 thus makes it an excellent case for further study of outflow precession.

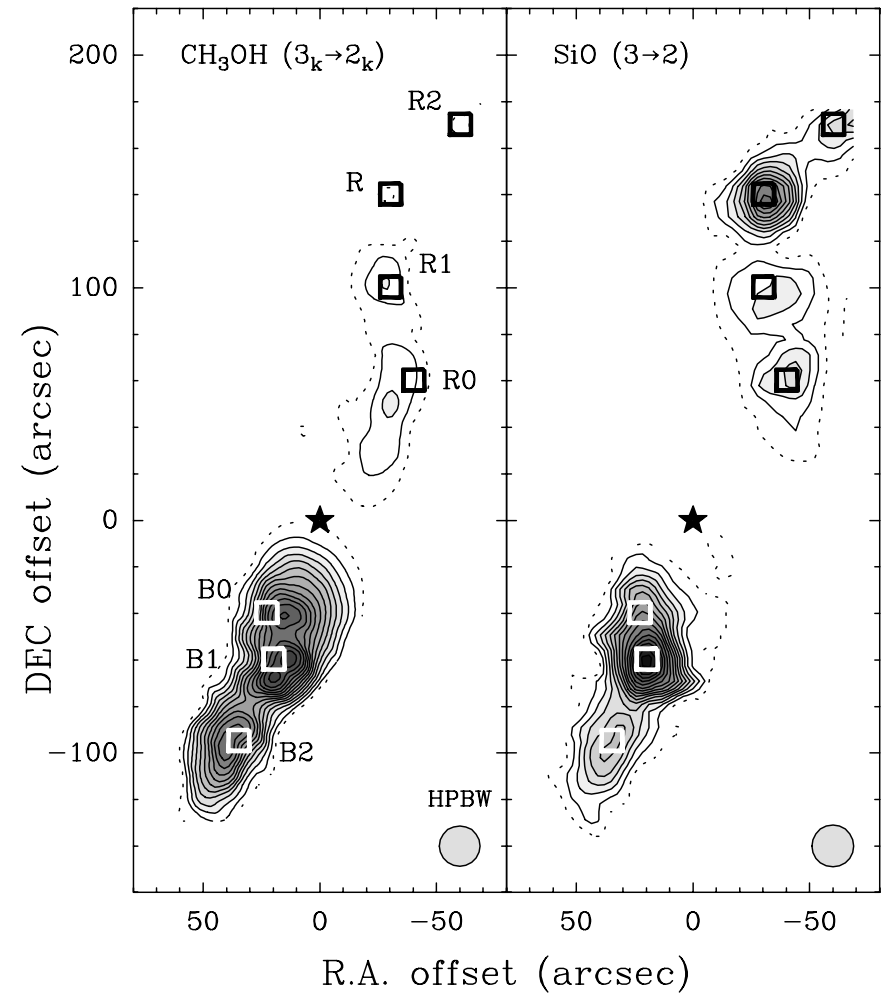

Fig. 10. Maps of the $\mathrm{CH}_{3} \mathrm{OH}$ and $\mathrm{SiO} J=3 \rightarrow 2$ integrated emission, showing both lobes of the L 1157 outflow. First contour and step are 5 and $1.5 \mathrm{~K} \mathrm{~km} \mathrm{~s}^{-1}$, respectively. Note the evident asymmetry of the emission, specially in the $\mathrm{CH}_{3} \mathrm{OH}$ map. The different condensations are not perfectly aligned, which indicates the precession of the outflow axis.

Although the precession model fits well the location of the bright outflow shocks, it does not fully explain the distribution of all accelerated gas. This can be seen from the right panel of Fig. 12, where the thickest sinusoidal line indicates the actual ejecta position (as in the left panel) and the thinner sinusoidal lines indicate past ejecta locations. If we assume a narrow jet in the isothermal limit (see, e.g., Masson \& Chernin 1993), the region swept up by the outflow will only extend over the region covered by thin sinusoidal waves. This makes it difficult to explain the weaker NE side of the red lobe and SW side of the blue lobe. We note, however, that this "anomalous" emission arises from material moving at the lowest outflow velocities (Fig. 2), so it is possible that represents material accelerated not directly by the driving jet, but by ambient gas already set into motion. Alternatively, a less collimated outflow component may coexist with the precessing jet. The smaller fraction of this "anomalous" gas, however, suggests that if this component exists, it represents a small perturbation to the jet driven flow.

Several mechanisms can explain the precession of an outflow jet. Eislöffel \& Mundt (1997) and Fendt \& Zinnecker (1998) have investigated the effects of the $J \times B$ forces or of a binary companion to the driving source, and either of these processes can be at work in L 1157 . The presence of a binary companion, in particular, is not 
completely ruled out by present data, as $\mathrm{mm}$ observations from Gueth et al. (1997) have a beam of about 3.5", which translates to more than $1000 \mathrm{AU}$ at the distance of 440 pc. Higher angular resolution observations are therefore needed to settle this point.

\subsection{Chemical stratification}

An important result from our observations is the prominent chemical segregation from the protostellar vicinity to the southern lobe of L 1157 and along the extent of this lobe (see Sect. 4.1). The LVG calculations with the molecules for which we have multiline observations show that, although the physical conditions only change moderately along the outflow, there are important differences in the physical conditions between the protostellar condensation and the shocked regions. The intensities of the narrow lines around the protostar are well explained as arising in a medium at a kinetic temperature of $13 \mathrm{~K}$ (as indicated by $\mathrm{NH}_{3}$ observations, see Bachiller et al. 1993) and a density of $\sim 10^{6} \mathrm{~cm}^{-3}$. Interferometric observations of a narrow $\mathrm{CH}_{3} \mathrm{OH}$ line component by Goldsmith et al. (1999), suggest higher excitation conditions, densities of $\sim 10^{7} \mathrm{~cm}^{-3}$, and temperatures of $\sim 50 \mathrm{~K}$, in the innermost region of the protostar, probably a disk of $<100$ AU radius.

The physical conditions in the shocked regions can be deduced from the broad emission lines observed toward the outflow lobes (see Sect. 4.1). In the blue lobe, when one moves from B0 to B2, the density changes from $n \sim 3$ to $6 \times 10^{5} \mathrm{~cm}^{-3}$ and the kinetic temperature from 40 to $80 \mathrm{~K}$. The sharp increase of the CO brightness temperature observed toward the blueshifted lobe (see Fig. 1) is clearly an effect of the gas heating produced by the shock (see also Umemoto et al. 1992; Bachiller et al. 1993; Hirano \& Taniguchi 2001).

As previously noted, the changes in physical conditions along the outflow lobes are insufficient to explain the large differences observed in the emission of the different molecular lines, so it is necessary to invoke the presence of strong gradients in the chemical composition of the shocked medium. BP97 already provided estimates of the abundance enhancement factors at the positions of the peaks. A extreme case is $\mathrm{CN}$ which appears to be enhanced by a factor of $\sim 100$ around B0, 30 around B1, and only $\sim 10$ around $\mathrm{B} 2$. $\mathrm{HCO}^{+}$exhibits a similar behavior: the enhancement factor decreases from $>100$ around B0 to values of $\sim 40$ near B1 and $\sim 20$ near B2. On the contrary, $\mathrm{SO}_{2}$ is not observed around $\mathrm{B} 0$, but appears to be enhanced by factors of $\sim 8-20$ around B1 and around B2. No significant changes are perceived in the abundances of $\mathrm{SiO}, \mathrm{CH}_{3} \mathrm{OH}$, and $\mathrm{H}_{2} \mathrm{CO}$ (which are enhanced by factors of $>10^{5}, \sim 400$, and $\sim 80$ toward B0, B1 and B2). We refer to BP97 for a complete list of the abundances estimated at the emission peaks.

It is very likely that the observed chemical gradients are related to the strong time dependence of the shock chemistry. As discussed in Sects. 3.1 and 5.1, the

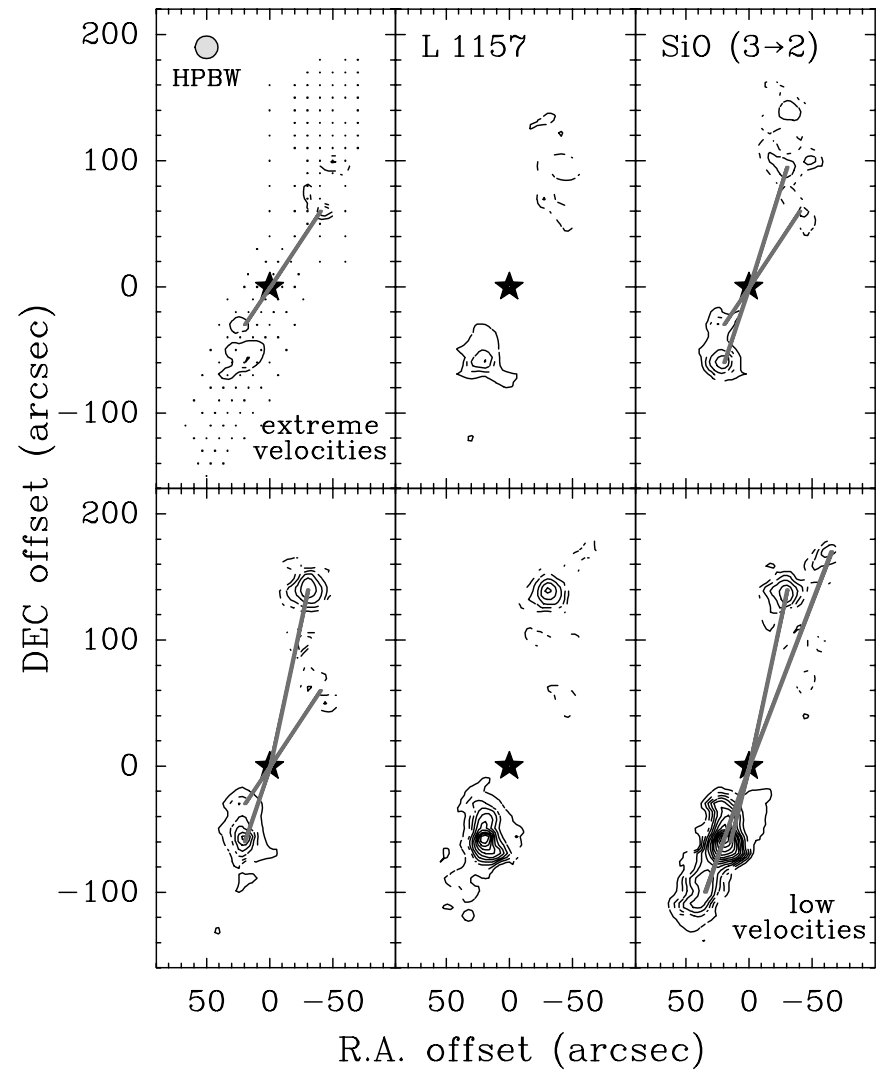

Fig. 11. SiO emission for the same intervals as in Fig. 2. First contour and step are $0.8 \mathrm{~K} \mathrm{~km} \mathrm{~s}^{-1}$ for the red lobe and 0.5 for the blue one. The thick lines (also shown in Fig. 2) represent different directions of the outflow.

successive emission peaks along the blueshifted outflow lobe correspond to successive ejection events. Given the differences in the velocities of the bulk emission at the successive peaks (see, for instance, Fig. 9), it results that B0, $\mathrm{B} 1$, and B2 correspond to events which are progressively older. In Sect. 3.1 we estimated the ages of B0, B1, and B2 to be 4500,7000 , and $15000 \mathrm{yr}$, respectively. The outflow axis can then be considered as a kind of time axis, and the chemical composition of the gas around B0, B1, and B2 could be considered as that of a shock which is rapidly evolving in time. We next discuss some of our results in the context of some recent time-dependent chemical theoretical models.

The chemistry of sulfur-bearing molecules in interstellar shocks has been described by Pineau des Forêts et al. (1993). More recently, Charnley (1997) has developed a model to explain the sulfur chemistry of hot cores at several temperatures which in principle can also be applied to a shock wave heating the gas. In both models the abundance of $\mathrm{H}_{2} \mathrm{~S}$ is first enhanced by several orders of magnitude. Pineau des Forêts et al. (1993) suggest that the passage of the shock wave induces the following sequence of ion-neutral reactions leading to the formation of $\mathrm{H}_{2} \mathrm{~S}: \mathrm{S}+\mathrm{H}_{3}^{+} \longrightarrow \mathrm{SH}^{+}+\mathrm{H}_{2} ; \mathrm{SH}^{+}+\mathrm{H}_{2} \longrightarrow \mathrm{H}_{2} \mathrm{~S}^{+}+\mathrm{H}$; $\mathrm{H}_{2} \mathrm{~S}^{+}+\mathrm{H}_{2} \longrightarrow \mathrm{H}_{3} \mathrm{~S}^{+}+\mathrm{H}$, finally followed by the dissociative recombination reaction: $\mathrm{H}_{3} \mathrm{~S}^{+}+\mathrm{e}^{-} \longrightarrow \mathrm{H}_{2} \mathrm{~S}+\mathrm{H}$. 
Neutral-neutral reactions with energy barriers could also be effective producing $\mathrm{H}_{2} \mathrm{~S}$ in the hot dense gas: $\mathrm{S}+$ $\mathrm{H}_{2} \longrightarrow \mathrm{SH} \longrightarrow \mathrm{H}_{2} \mathrm{~S}$. Charnley (1997) assumes that the sulfur content of grain mantles is predominantly in the form of $\mathrm{H}_{2} \mathrm{~S}$ (as suggested by near-IR spectroscopy toward massive protostars), and that these molecules are directly ejected to the gas phase. In any event, once present in the gas, $\mathrm{H}_{2} \mathrm{~S}$ gives rise to a series of hot phase chemical reactions to produce $\mathrm{SO}, \mathrm{SO}_{2}, \mathrm{CS}, \mathrm{OCS}$, and $\mathrm{H}_{2} \mathrm{CS}$. For instance, the models by Charnley (1997) predict a sharp decrease in the $\mathrm{H}_{2} \mathrm{~S}$ abundance after $\sim 10^{4}$ yr because oxidation with $\mathrm{O}$ and $\mathrm{OH}$ produces $\mathrm{SO}$ first and $\mathrm{SO}_{2}$ afterwards. These trends can in principle be compared to our observations of $\mathrm{L} 1157$. The estimate of the abundance ratio $\mathrm{SO}_{2} / \mathrm{H}_{2} \mathrm{~S}$ is 0.7 in peak $\mathrm{B} 1$, and 1.4 in peak $\mathrm{B} 2$ (see also $\mathrm{BP} 97$ ), and the $\mathrm{SO}_{2} / \mathrm{SO}$ ratio is $<1$ in $\mathrm{B} 1$ and $>1$ in $\mathrm{B} 2$. These values are in general agreement with the sequence $\mathrm{H}_{2} \mathrm{~S} \longrightarrow \mathrm{SO} \longrightarrow \mathrm{SO}_{2}$ predicted by the models. No significant differences are observed for CS between peak $\mathrm{B} 1$ and B2. This is also consistent with the trend found in the chemical models, in which CS remains more or less constant with time. Note also the dynamical timescales of shocks B1 and B2 are of the order of magnitude required by models.

$\mathrm{CH}_{3} \mathrm{OH}$ is expected to be released in the gas phase due to direct desorption from grain mantles. A model of the gas-phase chemistry in regions where ice mantles contaning $\mathrm{CH}_{3} \mathrm{OH}$ and other alcohols are evaporated from ice mantles was presented by Charnley et al. (1995). Once liberated, $\mathrm{CH}_{3} \mathrm{OH}$ is expected to disappear after a few $10^{4} \mathrm{yr}$ due to depletion back onto the grains, or to gas phase ion-molecule reactions, which produces complex organic molecules like $\mathrm{CH}_{3} \mathrm{OCH}_{3}$ and $\mathrm{HCOOCH}_{3}$ (see van Dishoeck \& Blake 1998, and references therein). The high abundances of $\mathrm{CH}_{3} \mathrm{OH}$ observed in the southern lobe of L 1157, comparable to those of hot cores like Ori-KL, means that the outflow is very young, and that the strong interaction between the shock wave and the quiescent medium is taking place right now. The enhancement of methanol does not last long once the underlying jet ceases or changes its direction (Sandford \& Allamandola 1993). This could explain the absence of this emission at farther distances in the northern lobe.

During the passage of the shock wave, sputtering of the grains can produce high abundances of gas phase $\mathrm{Si}$ or $\mathrm{Si}-$ bearing molecules in the post-shocked gas (Caselli et al. 1997). However, as $\mathrm{SiO}$ is a very refractory molecule, it depletes quickly onto the grains, with a timescale $\lesssim 10^{4} \mathrm{yr}$ (e.g. Mikami et al. 1992). Schilke et al. (1997) suggest that $\mathrm{SiO}$ can be destroyed more efficiently by reacting with $\mathrm{OH}: \mathrm{SiO}+\mathrm{OH} \longrightarrow \mathrm{SiO}_{2}+\mathrm{H}$, since $\mathrm{OH}$ is expected to be very abundant in the post-shock gas, where it is produced from the destruction of the water formed in the shock. Bergin et al. (1998) have found that water ice mantles are easily formed in the layer of water-rich gas processed by shocks with velocities in excess of $10 \mathrm{~km} \mathrm{~s}^{-1}$. Similarly to what happens with the water molecules, $\mathrm{SiO}_{2}$ is expected to condensate very quickly onto the grains mantles.
Models predict that $\mathrm{SiO}$ is transformed into $\mathrm{SiO}_{2}$ with a timescale of the order of $10^{4} \mathrm{yr}$, which is very similar to the timescale of $\mathrm{SiO}$ depletion. In any case (oxidation or depletion), $\mathrm{SiO}$ is expected to disappear from the gas phase in a few $\sim 10^{4}$ yr. This is in general agreement with the present observations. Similar conclusions were drawn from previous works in which the evolution of $\mathrm{SiO}$ in outflows was studied from an observational point of view (MartínPintado et al. 1992; Codella et al. 1999, and references therein).

The evolution of other molecular species is much more uncertain. Formaldehyde $\left(\mathrm{H}_{2} \mathrm{CO}\right)$ could rapidly be produced in gas phase if the abundance of $\mathrm{CH}_{3} \mathrm{OH}$ is high enough, as indicated by laboratory experiments (e.g. Bernstein et al. 1995). However, solid $\mathrm{H}_{2} \mathrm{CO}$ has been observed in the vicinity of young stellar objects (Schutte et al. 1996) suggesting that direct ejection from grain mantles is also a plausible mechanism to enhance its gas phase abundance. It seems anyway that the chemistries of $\mathrm{H}_{2} \mathrm{CO}$ and $\mathrm{CH}_{3} \mathrm{OH}$ are closely linked.

The cases of $\mathrm{CN}$ and $\mathrm{HCO}^{+}$are of high interest, since these molecules are observed to be more enhanced toward the youngest shocks. It seems that, after being enhanced in the shock, there are mechanisms that rapidly destroy these molecules. As suggested by BP97, CN could be efficiently destroyed by its reaction with atomic oxygen which is indeed expected to be abundant in the shocked region. But, unfortunately, the activation barrier of the reaction $\mathrm{CN}+$ $\mathrm{O} \longrightarrow \mathrm{CO}+\mathrm{N}$ is unknown. On the other hand, if water and methanol are abundant in the gas phase, as seems very likely in the shock, the ions $\mathrm{H}_{3} \mathrm{O}^{+}$and $\mathrm{CH}_{3} \mathrm{OH}_{2}^{+}$are expected to be formed quickly, and to produce $\mathrm{HCO}^{+}$by gas phase reactions. Afterwards, $\mathrm{HCO}^{+}$could be efficiently destroyed by dissociative recombination since the electronic abundance is presumably enhanced in the shocked region. The shock chemistry of $\mathrm{HCO}^{+}$has been modelled recently by Bergin et al. (1998) in the context of their detailed study of the evolution of $\mathrm{H}_{2} \mathrm{O}$ and $\mathrm{O}_{2}$ in shocks. Similar processes to those that control the $\mathrm{HCO}^{+}$abundance could account for the behaviour of $\mathrm{N}_{2} \mathrm{H}^{+}$which is not observed at high velocities.

In spite of the much effort which is being devoted during the last years to study theoretically the influence of shocks on the chemistry, the present discussion illustrates that many details remain poorly known. In fact, the classical gas-phase chemical problem is made even more complex in the presence of shock waves by the action of shocks on the mantles and cores of the dust grains. From a series of studies (e.g. Flower \& Pineau des Fôrets 1994, 1995; Flower et al. 1996) it is clear nowadays that sputtering, both thermal in high-velocity $J$-shocks and non-thermal in slower $C$-shocks, is responsible for injecting refractory and volatile species into the gas. Much chemical modelling which take into account these processes is being carried out recently (e.g. Pineau des Fôrets 1993; Schilke et al. 1997; Caselli et al. 1997; Bergin et al. 1998) and should continue in the future to provide details on the chemical processes taking place in shocks. 


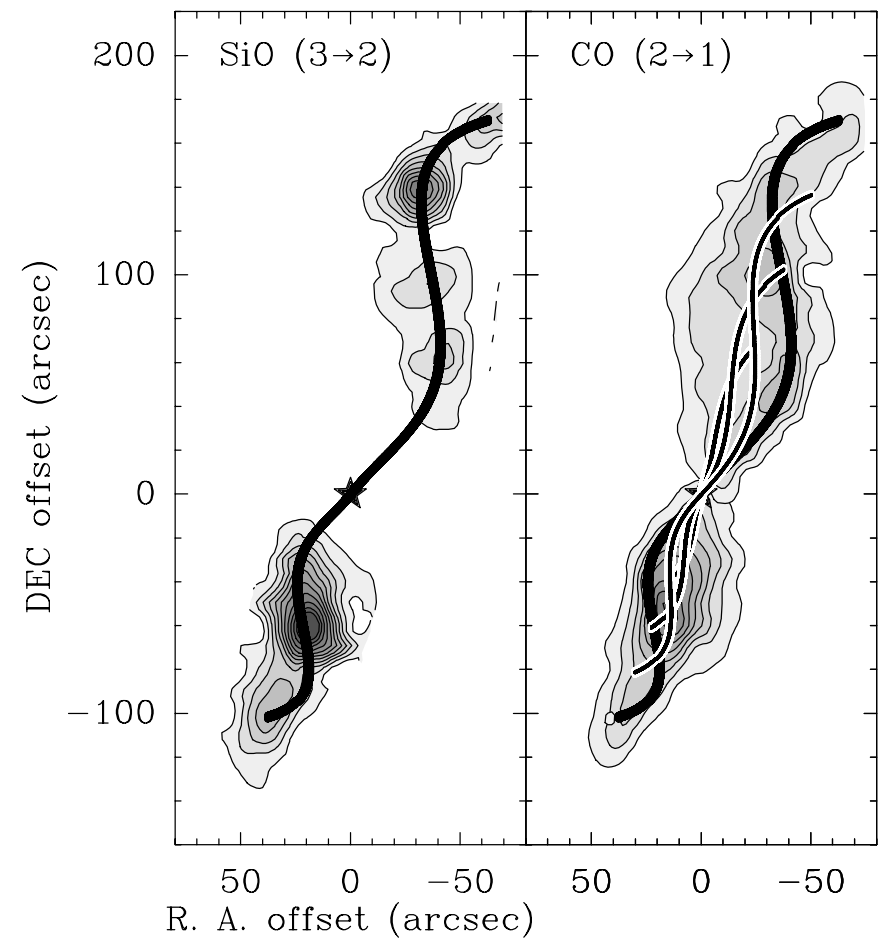

Fig. 12. Precession model predictions overlayed on the integrated $\mathrm{SiO}$ map of Fig. 10 (left) and the $\mathrm{CO}$ map of Fig. 1 (right). The thick sinusoidal line indicates the actual ejecta position, and the thin sinusoidal lines indicate ejecta positions at times equal to $0.80,0.60, \ldots$ the present time. The model parameters are jet velocity of $100 \mathrm{~km} \mathrm{~s}^{-1}$, period of $5000 \mathrm{yr}$, outflow age of $4000 \mathrm{yr}$ and a precession angle of $15^{\circ}$.

We finally point out that, as discussed by Houde et al. (2000a), the presence of a magnetic field is expected to cause a dramatic spatial segregation between neutrals and ions. This is because under some circumstances the magnetic forces on the ions can dominate over collisional processes generating a different pattern for the motion of ions and neutrals. This effect clearly depends on the relative orientation of the magnetic field and the bipolar outflow axis, and it requires a significant misalignement of both axis to be effective. But it seems to explain ion spectral line profiles with narrower line widths and lacking high velocity wings (when compared to the line profiles of the neutral species) similar to those that we observe for $\mathrm{N}_{2} \mathrm{H}^{+}$, and in a lesser extent for $\mathrm{HCO}^{+}$. Clearly, observations of different outflows presenting different geometric configurations are necessary to check the importance of this effect (Houde et al. 2000b, 2000c).

\subsection{1157 as prototype of "chemically active outflows"}

Bipolar outflows have been an essential element for the classification of young stellar objects (YSOs). As the circumstellar material around a YSO is dispersed by the action of its outflow, the YSO spectral energy distribution (SED) evolves systematically, and this allows to group
YSOs in Classes 0, I, II, and III (Lada 1991; André et al. 1993). However, it is clear nowadays that this classification is too schematic, and it results - for instance - that under the label of "Class 0" sources (the presumed youngest "sub-millimeter protostars") we find a rather hetereogeneous assemblage of YSOs. The inclination of the outflow (and that of the disk/protostar system) with respect to the line of sight makes difficult the distinction among objects which could be intrinsically different.

Indeed the main obstacle when studying the evolution of YSOs is the identification of a reliable time indicator. An attempt to describe YSO evolution in a unified way with main-sequence stars was done by Myers and Ladd (1993) by intruducing the "bolometric temperature" $T_{\text {bol }}$, but this parameter does not seem enough to classify Class

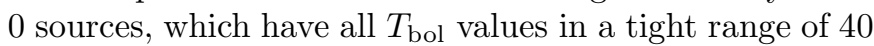
to $70 \mathrm{~K}$.

As discussed previously by Bachiller \& Tafalla (1999), outflows change dramatically during the first stages of the protostellar life, and could again be helpful to refine the classification of YSOs. These authors described a preliminary time sequence for outflows based on well known objects and in the chemical changes that are expected to induce in the surrounding molecular material. The first stage of this sequence is defined by jet-like outflows at extremely high velocities (EHV). These outflows are very highly collimated (collimation factor $>10$ ), and are composed of molecular "bullets" that appears as secondary EHV components in the spectra. The abundance of $\mathrm{SiO}$ appears highly enhanced at EHVs. The exciting source is a Class 0 protostar. Examples of this kind of outflows are L1448-mm (Bachiller et al. 1990), IRAS03282+3035 (Bachiller et al. 1991), and SVS13B (Bachiller et al. 1998).

At the end of the sequence described by Bachiller \& Tafalla (1999) there are the outflows from Class I sources in which a clear shell structure surrounding an evacuated cavity with a reflection nebula has been developed. Outflows from Class I sources are much less collimated and have a much lower mechanical power efficiency $\left(L_{\text {mech }} / L_{\text {bol }}\right)$ than those from Class 0 sources (Bontemps et al. 1996; Bachiller 1996). Class I outflows are associated with prominent Herbig-Haro objects and with observable kinematic perturbations in the surrounding dense core, but with no significant chemical anomalies. The prototype of such outflows is L1551/IRS5 (Moriarty-Schieven et al. 1989).

Between these two extreme types of young outflows, there is a kind of outflows for which we propose here the denomination of "chemically active outflows". These are less collimated and their terminal velocities are lower than in the phase before. There is no evidence for "bullets" in the spectra, but the maps show regions with large amounts of warm $(\sim 100 \mathrm{~K})$ gas. Very strong chemical anomalies are observed, with $\mathrm{SiO}, \mathrm{CH}_{3} \mathrm{OH}, \mathrm{H}_{2} \mathrm{CO}$, and $\mathrm{H}_{2} \mathrm{O}$ lines exhibiting prominent wings. The prototype of such outflows clearly is L1157, but other prominent example is BHR 71 (Bourke et al. 1997; Garay et al. 1998). The exciting source of these outflows is a Class 0, as in the phase 
before, but since the outflow seems more evolved (and presents a wider opening angle at the base) it is reasonable to assume that the driving sources of these outflows are slightly more evolved than those of the highly-collimated EHV flows.

There are outflows of similar collimation and velocities than the chemically active outflows, but with much smaller chemical anomalies, as if the shocks have already cooled down. Examples of such outflows are L483 (Tafalla et al. 2000) and L1527 (MacLeod et al. 1994). The exciting sources of these outflows seem in the transition from Class 0 to I (see e.g. Tafalla et al. 2000) suggesting that these are the link from the "chemically active outflows" to the Class I outflows.

This classification scheme needs to be more firmly established by the means of observations of a complete sample of outflows in different evolutionary stages. Such a study is under way, and we expect to provide additional results in the next future. In any event, what results clear from the previous discussion is that the chemical anomalies induced by outflows provide a powerful tool to refine the classification of young protostars. We finally point out that $\mathrm{L} 1157$, as the most conspicuous representative of chemically active outflows, also provides a unique opportunity to study chemical processes that can be compared to those operating in other astrochemical circumstances, such as hot cores, photo-dominated regions, or comets. For instance, Bocklee-Morvan et al. (2000) from a comparison of the abundances in L 1157 with those found in comet C/1995 Hale-Bopp were able to study the link between the cometary and the interstellar material. A more detailed investigation of the molecular abundances in L 1157, including very rare species, is underway and will be reported elsewhere.

\section{Summary and conclusions}

We have conducted detailed mapping observations of the L 1157 outflow in several molecular lines including CO. The new data have provideded important results on the structure and chemistry of the outflow that are summarised below.

1. New CO maps have enabled us to characterise the physical properties of the molecular outflow. The outflow has a total length of $0.8 \mathrm{pc}$ and contains a mass of $\sim 0.6 M_{\odot}$. We found that there are notable asymmetries between the northern and southern lobe in terms of their sizes and physical parameters, but the morphology clearly display a repetitive S-shaped point-symmetry indicating the presence of an underlying precessing jet.

2. We have found evidence for 3 or 4 independent episodes of mass ejection from the central source. The kinematical timescale of the oldest episodes is $\sim 15000 \mathrm{yr}$, and the time elapsed between successive episodes is a few $10^{3} \mathrm{yr}$.

3. From several maps in different molecular spectral lines we conclude that those of $\mathrm{C}_{3} \mathrm{H}_{2}, \mathrm{DCO}^{+}$and $\mathrm{N}_{2} \mathrm{H}^{+}$ trace only the central condensation (along the axis of a hypothetical disk) and are not observed in the outflow. These are the best lines to further study the structure and kinematics of the material surrounding the protostar. Such a study would require higher angular and spectral resolution than that of the data presented here.

4. A chemical study of the blueshifted lobe show strong gradients in the distribution of the emission from different molecules. The emission gradients are due to chemical stratification and not only to excitation effects. The chemical stratification is shown to be a consequence of the chemical evolution of the outflowing gas. These observations, together with other recent studies of Class 0 sources (Blake et al. 1994, 1995; van Dishoeck et al. 1995), illustrate the potential of the time-variable chemistry to study the first stages of evolution of bipolar outflows.

5. Given the resolution of the present maps, and the rapidity with which the chemistry is expected to evolve, we cannot study in detail if different molecules are generated in different parts of the bow-shocks. However some trends are observed: $\mathrm{SiO}$ seems to be produced at the shock head or at the precursor, whereas other molecules like $\mathrm{CH}_{3} \mathrm{OH}$, $\mathrm{HCN}$ or $\mathrm{HC}_{3} \mathrm{~N}$ could be produced at the shock wings by turbulent entrainment. The chemical and spatial segregation has to be studied in individual bow shocks. This requires mapping of the blue lobe of L 1157 with high angular resolution in different molecular lines that cover a wide range of the chemical processes operating at the shocks.

6. The observations of $\mathrm{SiO}$ and broad $\mathrm{NH}_{3}$ emission (Bachiller et al. 1993), $\mathrm{CO}$ (Zhang et al. 2000) and $\mathrm{H}_{2}$ emission (Davis \& Eislöffel 1995) of the red lobe clearly indicate that shocks are present in the northern lobe also. However, most molecular lines present weaker intensities than toward the south lobe suggesting that important physical and chemical differences exist between both lobes. Such differences could be due to the structure of the molecular cloud before the star formation activity started.

7. L 1157 can be considered as the prototype of a particular category of outflows from Class 0 sources for which we propose the denomination of "chemically active outflows". These outflows seem to be more evolved than highly-collimated outflows with "bullets" (such as L1448$\mathrm{mm}$ ), but less evolved than other outflows from objects which are in transition to the Class I (such as L 483).

Acknowledgements. This paper benefited from helpful comments and suggestions of an anonymous referee. The authors are also grateful to Drs. Asunción Fuente and Claudio Codella for extensive and fruitful discussions. This research have been partially supported by Spanish DGES grants PB96-0104 and AYA2000-927.

\section{References}

André, P., Ward-Thompson, D., \& Barsony, M. 1993, ApJ, 406, 122

Avery, L. W., \& Chiao, M. 1996, ApJ, 463, 642 
Bachiller, R., Cernicharo, J., Martín-Pintado, J., Tafalla, M., \& Lazareff, B. 1990, A\&A, 231, 174

Bachiller, R., Guilloteau, S., Gueth, F., et al. 1998, A\&A, 339, L49

Bachiller, R., Martín-Pintado, J., \& Planesas, P. 1991, A\&A, 251,639

Bachiller, R., Martín-Pintado, J., \& Fuente, A. 1993, ApJ, 417, L45

Bachiller, R. 1996, ARA\&A, 34, 111

Bachiller, R., \& Pérez Gutiérrez, M. 1997, ApJ, 487, L93 (BP97)

Bachiller, R., Codella, C., Colomer, F., Liechti, S., \& Walmsley, C. M. 1998, A\&A, 335, 266

Bachiller, R., \& Tafalla, M. 1999, The Origin of Stars and Planetary Systems, ed. C. J. Lada, \& N. Kylafis, NATO Sci. Ser. (Kluwer Acad Pub), 227-266

Bally, J., Devine, D., \& Reipurth, B. 1996, ApJ, 473, L49

Bence, S. J., Richer, J. S., \& Padman, R. 1996, MNRAS, 279, 866

Bergin, E. A., Melnick, G. J., \& Neufeld, D. A. 1998, ApJ, 499, 777

Bernstein, M. P., Sandford, S. A., Allamandola, L. J., Chang, S., \& Scharberg, M. A. 1995, ApJ, 454, 327

Bocklee-Morvan, D., Lis, D. C., Wink, J. E., et al. 2000, A\&A, 353,1101

Bontemps, S., André, P., Terebey, S., \& Cabrit, S. 1996, A\&A, 297, 98

Bourke, T. L., Garay, G., Lehtinen, K. K., et al. 1997, ApJ, 1997, 476, 781

Blake, G. A., van Dishoeck, E. F., Jansen, D. J., Groesbeck, T. D., \& Mundy, L. G. 1994, ApJ, 428, 680

Blake, G. A., Sandell, G., van Dishoeck, E. F., et al. 1995, ApJ, 441,689

Cabrit, S., \& Bertout, C. 1990, ApJ, 348, 530

Cabrit, S., Couturier, S., André, P., et al. 1998, Star formation with the Infrared Space Observatory, ed. J. L. Yun, \& R. Liseau, ASP Conf. Ser., 132, 326

Caselli, P., Hartquist, T. W., \& Havnes, O. 1997, A\&A, 322, 296

Codella, C., Bachiller, R., \& Reipurth, B. 1999, A\&A, 343, 585

Charnley, S. B., Kress, M. E., Tielens, A. G. G. M., \& Millar, T. J. 1995, ApJ, 448, 232

Charnley, S. B. 1997, ApJ, 481, 396

Cummings, S. E., Linke, R. A., \& Thaddeus, P. 1986, ApJSS, 60,819

Davis, C. J., \& Eislöffel, J. 1995, A\&A, 300, 851

Eislöffel, J., \& Mundt, R. 1997, AJ, 114, 280

Fendt, C., \& Zinnecker, H. 1998, A\&A, 334, 571

Flower, D. R., \& Pineau des Fôrets, G. 1994, MNRAS, 268, 724

Flower, D. R., \& Pineau des Fôrets, G. 1995, MNRAS, 275, 1049

Flower, D. R., Pineau des Fôrets, G., Field, D., \& May, P. W. 1996, MNRAS, 280, 447

Garay, G., Köhnenkamp, I., Bourke, T. L., Rodríguez, L. F., \& Lehtinen, K. K. 1998, ApJ, 509, 768
Goldsmith, P. F., Langer, W. D., \& Velusamy, T. 1999, ApJ, 519, L173

Gómez, M., Kenyon, S. J., \& Whitney, B. A. 1997, AJ, 114, 265

Gueth, F., Guilloteau, S., \& Bachiller, R. 1996, A\&A, 307, 891 (GGB)

Gueth, F., Guilloteau, S., Dutrey, A., \& Bachiller, R. 1997, A\&A, 323, 943

Gueth, F., Guilloteau, S., \& Bachiller, R. 1998, A\&A, 333, 287

Hirano, N., \& Taniguchi, Y. 2001, ApJ, in press

Houde, M., Bastien, P., Peng, R., Phillips, T. G., \& Yoshida, H. 2000a, ApJ, 536, 857

Houde, M., Peng, R., Phillips, T. G., Bastien, P., \& Yoshida, H. 2000b, ApJ, 537, 245

Houde, M., Phillips, T. G., Bastien, P., Peng, R., \& Yoshida, H. 2000c, ApJ, in press

Lada, C. J. 1991, The Physics of Star Formation and Early Evolution, ed. C. J. Lada, \& N. D. Kylafis (Kluwer: Dordrecht)

MacLeod, J., Avery, L., Harris, A., \& Tacconi, L. 1994, JCMT Newslett., September/October, 46

Margulis, M., \& Lada, C. J. 1985, ApJ, 299, 925

Martín-Pintado, J., Bachiller, R., \& Fuente, A. 1992, A\&A, 254,315

Mikami, H., Tomofumi, U., Yamamoto, S., \& Saito, S. 1992, ApJ, 392, L87

Moriarty-Schieven, G. H., Snell, R. L., \& Hughes, V. A. 1989, ApJ, 347, 358

Pineau des Forêts, G., Roueff, E., Schilke, P., \& Flower, D. 1993, MNRAS, 262, 915

Raga, A. C., \& Cabrit, S. 1993, A\&A, 278, 267

Reipurth, B., \& Raga, R. 1999, The Origin of Stars and Planetary Systems, ed. C. J. Lada, \& N. Kylafis, NATO Sci. Ser. (Kluwer Acad Pub), 267

Sandford, S. A., \& Allamandola, L. J. 1993, ApJ, 417, 815

Schilke, P., Malmsley, C. M., Pineau des Forêts, G., \& Flower, D. 1997, A\&A, 321293

Schutte, W. A., Gerakines, P. A., Geballe, T. R., van Dishoeck, E. F., \& Greenberg, J. M. 1996, A\&A, 309, 633

Stahler, S. W. 1994, ApJ, 422, 616

Tafalla, M., \& Bachiller, R. 1995, ApJ, 443, L37

Tafalla, M., Myers, P. C., Mardones, D., \& Bachiller, R. 2000, A\&A, 359, 967

Turner, B. E., Chan, K.-W., Green, S., \& Lubowich, D. A. 1992, ApJ, 399, 114

Umemoto, T., Iwata, T., Fukui, Y., et al. 1992, ApJ, 392, L83

Umemoto, T., Mikami, H., Yamamoto, S., \& Hirano, N. 1999, ApJ, 525, L105

van Dishoeck, E. F., Blake, G. A., Jansen, D. J., \& Groesbeck, T. D. 1995, ApJ, 447, 760

van Dishoeck, E. F., \& Blake, G. A. 1998, ARA\&A, 36, 317

Wilson, T. L., \& Rood, R. 1994, ARA\&A, 32, 191

Zhang, Q., Ho, P. T. P., Wright, M. C. H., \& Wilner, D. J. 1995, ApJ, 451, L71

Zhang, Q., Ho, P. T. P., \& Wright, M. C. H. 2000, AJ, 119, 1345 